\title{
Reduction of Boundary Value Problem to Possio Integral Equation in Theoretical Aeroelasticity
}

\author{
A. V. Balakrishnan ${ }^{1}$ and M. A. Shubov ${ }^{2}$ \\ ${ }^{1}$ Electrical Engineering Department, University of California, Los Angeles, CA 90095, USA \\ ${ }^{2}$ Department of Mathematics and Statistics, University of New Hampshire, Durham, NH 03824, USA
}

Correspondence should be addressed to M. A. Shubov, marianna.shubov@euclid.unh.edu

Received 9 January 2008; Accepted 25 May 2008

Recommended by Bernard Geurts

The present paper is the first in a series of works devoted to the solvability of the Possio singular integral equation. This equation relates the pressure distribution over a typical section of a slender wing in subsonic compressible air flow to the normal velocity of the points of a wing (downwash). In spite of the importance of the Possio equation, the question of the existence of its solution has not been settled yet. We provide a rigorous reduction of the initial boundary value problem involving a partial differential equation for the velocity potential and highly nonstandard boundary conditions to a singular integral equation, the Possio equation. The question of its solvability will be addressed in our forthcoming work.

Copyright (C) 2008 A. V. Balakrishnan and M. A. Shubov. This is an open access article distributed under the Creative Commons Attribution License, which permits unrestricted use, distribution, and reproduction in any medium, provided the original work is properly cited.

\section{Introduction}

The present paper is the first in a series of three works devoted to a systematic study of a specific singular integral equation that plays a key role in aeroelasticity. This is the Possio integral equation that relates the pressure distribution over a typical section of a slender wing in subsonic compressible air flow to the normal velocity of the points on a wing surface (downwash). First derived by Possio [1], it is an essential tool in stability (wing flutter) analysis. In spite of the fact that there exists an extensive literature on numerical analysis of the Possio (or modified Possio) equation, its solvability has never been proved rigorously. In our study, we focus on subsonic compressible flow. The problem of a pressure distribution around a flying wing can be reduced to a problem of velocity potential dynamics. Having a pressure distribution over a flying wing, one can calculate forces and moments exerted on a wing due to the air flow, which is an extremely important component of a wing modeling. Mathematically, the problem can be formulated in the time domain in the form of a system of nonlinear 


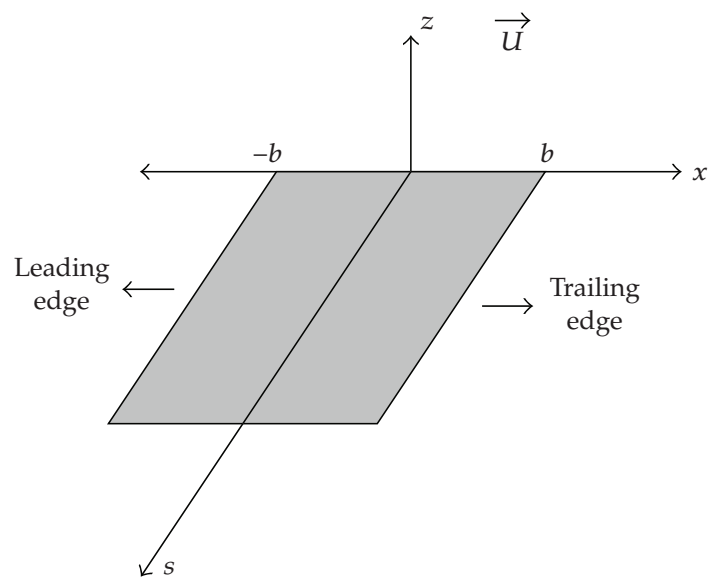

Figure 1: Wing structure beam model.

integrodifferential equations in a state space of a system (evolution-convolution equations). (As illustrating example for the case of an incompressible air flow, see [2-4].) So, the problem of stability involves as an essential part a nonlinear equation governing the air flow. Assuming that the flow is subsonic, compressible, and inviscid, we will work with the linearized version together with the Neumann-type boundary condition on a part of the boundary. It can be cast equivalently as an integral equation, which is exactly the Possio integral equation. Our main goal is to prove the solvability of this equation using only analytical tools. We should mention here that most of the research is currently done by different numerical methods, for example, $[5,6]$, and very few papers on analytical treatment of the Possio equation are available $[7,8]$. In essence, the partial differential equations are approximated by ordinary differential equations for both the structural dynamics and aerodynamics. However, it is important to retain full continuous models. It is the Possio integral equation that is the bridge between Lagrangian structural dynamics and Eulerian aerodynamics. In particular, using the solution of the Possio equation, one can calculate the aerodynamic loading for the structural equations. As the simplest structural model (Goland model $[9,10]$ ), let us consider a uniform rectangular beam (see Figure 1) endowed with two degrees of freedom, plunge and pitch. Let the flow velocity be along the positive $x$-axis with $x$ denoting the cord variable, $-b \leqslant x \leqslant b$; let $y$ be the span or length variable along the $y$-axis, $0 \leqslant y \leqslant l$. Let $h$ be the plunge, or bending, along $z$-axis; let $\theta$ be the pitch, or torsion angle, about the elastic axis located at $x=a b$, where $0<a<1$ and $a b$ is the distance between the center-line and the pitch axis; let $X(y, t)=(h(y, t), \theta(y, t))^{T}$ (the superscript " $T$ " means the transposition). Then the structural dynamics equation is

$$
\mathbf{M} X_{t t}(y, t)+\mathbf{K X}(y, t)=(\mathbb{L}(y, t), \mathbb{M}(y, t))^{T},
$$

where $\mathbf{M}$ is the mass-inertia matrix and $\mathbf{K}$ is the stiffness differential operator, $\mathbf{K}=$ $\operatorname{diag}\left\{E I\left(\partial^{4} / \partial y^{4}\right),-G J\left(\partial^{2} / \partial y^{2}\right)\right\}$, with $E I$ and $G J$ being the bending and torsion stiffness, respectively. $\mathbb{L}(y, t)$ and $\mathbb{M}(y, t)$ are the aerodynamic lift and moment about the elastic axis. System (1.1) is considered with the boundary conditions $h(0, t)=h^{\prime}(0, t)=\theta(0, t)=0 ; h^{\prime \prime}(l, t)=$ $h^{\prime \prime \prime}(l, t)=\theta^{\prime}(l, t)=0$. Certainly the structure model can be and has been extended to several degrees of freedom (but still linear) or to nonlinear models [11, 12]. For example, in [12] 
complete and general nonlinear theory with particular emphasis given to the fundamentals of the nonlinear behavior has been developed. The theory is intended for applications to long, straight, slender, homogeneous isotropic beams with moderate displacements, and is accurate up to the second order provided that the bending slopes and twist are small with respect to unity. Radial nonuniformities (mass, stiffness, twist), cordwise offsets of the mass centroid and tension axis from the elastic axis, and wrap of the cross-section are included into the structural equations. The nonlinearities can be important in determining the dynamic response of cantilever blades, and they are especially important in determining the aeroelastic stability of torsionally flexible blades. One can also add to (1.1) a control term as in [13,14]. However, the emphasis in the present paper is on aerodynamics - by far the most complicated part—and the structure interaction, specifically, on force and moment terms in (1.1).

Now we mention that the distinguishing feature of the aerodynamic model is that the air flow is assumed to be nonviscous. The flutter instability phenomena are captured already in nonviscous flow. Hence, the governing field equation is no longer the Navier-Stokes equation but the Euler full potential equation, and the main assumption is that the entropy is constant. As a result, the flow is curl-free and can be described in terms of a velocity potential. The unknown variable in the Possio equation is the velocity potential. Let us show that the velocity potential yields explicit expressions for the lift and moment from (1.1) (see, e.g., [9]).

The aerodynamic lift and moment (per unit length) are given by the formulas

$$
\mathbb{L}(y, t)=\int_{-b}^{b} \delta p d x, \quad \mathbb{M}(y, t)=\int_{-b}^{b}(x-a) \delta p d x,
$$

where $p$ is pressure and $\delta p=p(x, y, 0+, t)-p(x, y, 0-, t), 0 \leqslant y \leqslant l,|x|<b$. As already mentioned, the velocity potential $\varphi(x, y, z, t)$ satisfies the Euler full potential equation [8]. If one can derive a representation for the potential $\varphi$, then the following formula can be used for pressure calculation:

$$
p(x, y, z, t)=p_{\infty}\left(1+\frac{\gamma-1}{a_{\infty}^{2}}\left(\frac{U^{2}}{2}-\frac{\partial \varphi}{\partial t}-\frac{\nabla \varphi \cdot \nabla \varphi}{2}\right)\right)^{\gamma /(\gamma-1)}
$$

where $p_{\infty}=\rho^{2} a_{\infty}^{2} \gamma^{-1}, a_{\infty}$ is the far-field speed of sound, $\rho$ is the air density, and $\gamma>1$ is the adiabatic constant; $U$ is a speed of a moving wing. So knowing the velocity potential $\varphi$, we immediately obtain $\delta p$ and thus lift $\mathbb{L}$ and moment $\mathbb{M}$.

Any aeroelastic problem breaks into two parts. First, it is a field equation (for a linearized version of the field equation for a velocity potential $\varphi$, see (2.2)) with the boundary conditions, that is, (a) flow tangency condition (see (2.3)), (b) Kutta-Joukowski condition (see (2.5)), and (c) far-field conditions (see (2.6)). It is the flow tangency condition that establishes the connection between the structural and aerodynamical parts since the downwash function $w_{a}$ of (2.3) is expressed explicitly in terms of the plunge $h$ and pitch $\theta$. We mention here that the unique feature of the aerodynamic boundary conditions is that we have the Neumann condition only on a part of the boundary $z=0$, that is, only on the structure, and a related but different condition on the rest of the boundary, which is purely aerodynamic (not involving the structure dynamics). The second part involves the structure state variables via flow tangency condition (2.3) and more importantly the lift and moment.

Summarizing all of the above, one can see that the entire aeroelastic problem (structure and aerodynamics) becomes in general a nonlinear convolution (due to the expressions for the lift and moment) and/or evolution equation in terms of the structure state variables, whose stability with respect to the parameter $U$ is then the "flutter problem" one has to resolve. 
Dealing with the "flutter problem," one cannot proceed without mentioning that it was an important topic of famous Th.Theodorsen research (see, e.g., [15]). The determination of pressure distribution and aerodynamic loads on an airfoil exposed to a two-dimensional stream of incompressible fluid flow was a central problem in aeronautics of the early 1930's. Flutter was first encountered on tail-planes and wings during World War I, but rigorous theory for its prediction took many years to develop. The greatest challenge was to supply aerodynamic terms for the governing equations. This was the strongest motivation for research on air-loads experienced by wings and airfoils performing time-dependent motions. It was recognized that the first step would be to adapt the methods of the "thin-airfoil theory" so as to account for phenomena such as small oscillations normal to the directions of flight and impulsive changes of angle of attack. The main feature of the analytical scheme in Theodorsen's technical report [15] (which is now a classical paper) was related to the Joukowski transformation between parallel-stream flows past a circle and a zero-thickness flat plate. Even though this looks as oversimplification, the author of $T R-496$ (see [15]) knew that within the framework of thin-airfoil theory, the steady-flow problems of thickness and camber could be rigorously separated from the unsteady case, on which he focused. He correctly enforced the Kutta condition in the presence of infinite wake of trailing vortices. The main discovery of this report is a set of complex frequency-response functions connecting vertical translation (or bending) and angle of attack (or torsion) as "inputs" with unsteady lift and pitching moment as "outputs." The most important result of the entire investigation was that regardless of the nature of the small oscillation or of the "output" quantity to be found, only a single transcendental function appears in their relationship. It is the well-known "Theodorsen function"

$$
\mathcal{C}(k)=F(k)+i G(k)=\frac{H_{1}^{(2)}(k)}{H_{1}^{(2)}(k)+i H_{0}^{(2)}(k)},
$$

with $H_{0,1}^{(2)}(k)$ being the Hankel functions of the zero or first order, respectively, and of the second kind. This exact flutter solution including results for control surfaces has had a keystone role in the flutter analysis. It is interesting to note that Theodorsen function $\mathcal{C}(k)$ occurs in the theory of propulsion of birds and fish as well. With the aerodynamic terms constructed by adapting the formulas and ideas in $T R-496$ [15], it becomes possible to predict critical "flutter boundaries" quite accurately.

One of the most complete and important sources related to contemporary status of aeroelasticity is found in [16], where theoretical methods are combined with experimental and numerical results providing better understanding of the theory and its limitations. As demonstrated in [16], much of the theoretical and experimental developments can be applied to different engineering areas, and a common language can be used for explanation of different phenomena. Even though historically the entire field of aeroelasticity has centered in aeronautical applications, now the applications are found in civil engineering (on flows about bridges and tall buildings, see [16, Chapter 6] and [17]); in mechanical engineering (on flows about turbomachinery blades and fluid flows in flexible pipes, see [16, Chapters $3,7,8$, and 12] and [18]); in nuclear engineering (on flows about fuel elements and heat exchanger vanes). Moreover, aeroelasticity plays a crucial role in the development of new aerospace systems such as unmanned air vehicles (UAVs) [19, 20].

Before we turn to the description of the results of the present paper, we would like to mention earlier efforts to use integral transformations in order to calculate lift and pitching 
moment on a wing of given shape. Venters [21] presents a lifting surface theory for steady incompressible flows based on a shear flow rather than a potential flow model. The theory developed in [21] is intended to account for the boundary layer. The method of Fourier transforms is used to evaluate the pressure on a surface of infinite extent and arbitrary contour. The research initiated in [21] is continued in $[22,23]$, where a general theory of planar disturbances of inviscid parallel shear flows has been developed. This theory has been successfully applied to such problems as the generation of waves at a free surface, the interaction of a boundary layer with a flexible wall, the flow about a wing in or near a jet or wake, and the influence of the main boundary layer of a wing on control surface effectiveness.

Now we briefly describe the content of the present paper. In Section 2, we give a rigorous formulation of an initial boundary value problem for a partial differential equation for a disturbance potential (see (2.2)). We discuss the $L_{p}$-space, $1<p<2$, setting for the equation and the boundary conditions. In Section 3, we present a new form for the initial boundary value problem by applying two integral transformations, which yields Laplace transform in time variable and Fourier transform in $x$-variable of the unknown function. Such a double transform allows us to give the first version of the Possio equation (see (3.22)). Evidently, (3.22) is only the convenient initial point for the next move, that is, eliminating the Fourier transform representation. Section 4, being just a technical result, is very important. In this section, we prepare the main equation from (3.22) for Mikhlin multiplier theory application. In Section 5, we transform (3.22) to a singular integral equation, which is singular in more than one sense. Brief discussion and conclusions are given in Section 6.

In the conclusion of the introduction, we briefly outline the original version of the Possio equation [1], and provide some justification that a different version of it, derived and studied in the aforementioned series of our papers, is more convenient for analytical investigation.

The derivation of the original Possio integral equation [1], that can be found in [9], is based on representing the airfoil with a sheet of acceleration potential doublets along the projection of the airfoil. The doublet is obtained from a simple solution of (6.80) in [9] (which coincides with (2.8) of the present paper) known as a source pulse. Following the steps presented in [9], one obtains a sinusoidally pulsating doublet of some frequency $\omega$. After nontrivial calculations, one arrives at the Possio integral equation of the form

$$
\overline{w_{a}(x)}=-\frac{\omega}{\rho_{\infty} u^{2}} \text { P.V. } \int_{-b}^{b} \overline{\Delta p_{a}} \Re\left(M, \frac{k(x-\xi)}{b}\right) d \xi, \quad|x|<b .
$$

The problem is to find an operator, which is inverse to the above integral operator understood as a Cauchy principal value integral. The kernel function $\mathfrak{R}$ is extremely complicated and is given explicitly by the following formula:

$$
\begin{aligned}
\Re=\frac{1}{4 \sqrt{1-M^{2}}}\{ & \exp \left(i \frac{k M^{2}(x-\xi)}{b\left(1-M^{2}\right)^{2}}\right)\left[i M \frac{|x-\xi|}{x-\xi} H_{1}^{(2)}\left(\frac{k M|x-\xi|}{b\left(1-M^{2}\right)}\right)-H_{0}^{(2)}\left(\frac{k M|x-\xi|}{b\left(1-M^{2}\right)}\right)\right] \\
+ & i\left(1-M^{2}\right) \exp \left(i \frac{k(x-\xi)}{b}\right)\left[\frac{2}{\pi \sqrt{1-M^{2}}} \ln \left(\frac{1+\sqrt{1}-M^{2}}{M}\right)\right. \\
& \left.\left.+\int_{0}^{k(x-\xi) / b\left(1-M^{2}\right)} e^{i v} H_{0}^{(2)}(M|v|)\right]\right\},
\end{aligned}
$$


where $M$ is the Mach number, $k=\omega b / U$ is the reduced frequency, $H_{j}^{(2)}, j=0,1$, is the Hankel function of the second kind and of the zero or first order, respectively. By examination, one can see that (1.5) is very complicated not only because its kernel (1.6) contains special functions with nonstandard arguments, but also because it is really difficult to describe explicitly the nature of singularities. Contrary to (1.5), in the equation that we study in our series of papers, we can precisely formulate the nature of singularities. Namely, in our version, the Possio integral equation is singular by two reasons: (a) it contains singular integral operations, that is, the finite Hilbert transformation and its specific "inverse;" (b) one integral equation contains integral operators being different by their nature, that is, the finite Hilbert transformation, Volterra integral operator, and an integral operator with the degenerate kernel. Each type of integral operation requires its own approach, which makes the problem such a challenge. Regarding the Possio integral equation in its original form, C. Possio himself attempted to solve the equation using numerical integration technique. He presented an unknown function in the form of a series: $\overline{\Delta p_{a}}=A_{0} \cot (\theta / 2)+\Sigma_{n \geqslant 1} A_{n} \sin (n \theta), \xi / \beta=\cos \theta$. However, this substitution does not permit a straightforward inversion, and also convergence of the series is not always rapid. Many attempts have been made to develop a good numerical scheme for inversion of the integral in the Possio equation, but rigorous proof of the solvability of the equation has never been produced. In our series of papers, we are addressing the problem of the unique solvability using rigorous analytical tools.

\section{Aerodynamic field equation}

In this section, we start with the initial boundary value problem for a partial differential equation, which is known in the literature as the "small disturbance potential field equation" for subsonic inviscid compressible flow [8]. We assume that the air flow is around a large aspect-ratio planar wing, which means that the dependence on the span variable along the wing is neglected. The wing is then reduced to a "typical section" or a "chord."

We will use the following notations: $U$ is the free-stream velocity; $a_{\infty}$ is the sound speed; $M=U / a_{\infty}$ is the Mach number $0<M \leqslant 1$.

The velocity potential of the airflow is given by the expression

$$
U x+\varphi(x, z, t), \quad-\infty<x<\infty, 0 \leqslant z<\infty, t>0,
$$

where $U x$ is the free-stream velocity potential, and $\varphi$ is a small perturbation of the velocity potential. The disturbance potential $\varphi$ satisfies the following linearized field equation [7]:

$$
\begin{array}{r}
\varphi_{t t}(x, z, t)+2 M a_{\infty} \varphi_{t x}(x, z, t)=a_{\infty}^{2}\left(1-M^{2}\right) \varphi_{x x}(x, z, t)+a_{\infty}^{2} \varphi_{z x}(x, z, t), \\
-\infty<x<\infty, 0 \leqslant z<\infty, t>0 .
\end{array}
$$

Together with this equation, we introduce the following boundary conditions.

(1) The flow tangency (or nonseparable flow) condition is

$$
\left.\frac{\partial}{\partial z} \varphi(x, z, t)\right|_{z=0}=w_{a}(x, t), \quad|x|<b,
$$

where $w_{a}(x, t)$ is the given normal velocity of the wing (the downwash); $b$ is a size of a "half chord." We note that condition (2.3) is a nonhomogeneous Neumann condition prescribed only on a part of the boundary, $z=0$. 
(2) The Kutta-Joukowski conditions. To formulate these conditions, we need one more function, that is, the acceleration potential defined by

$$
\psi(x, z, t)=\varphi_{t}(x, z, t)+U \varphi_{x}(x, z, t) .
$$

The conditions below reflect the following physical situation: pressure off the wing and at the trailing edge must be equal to zero. These conditions are

$$
\psi(x, 0, t)=0 \quad \text { for }|x|>b, \quad \lim _{x \rightarrow b-0} \psi(x, 0, t)=0 .
$$

(3) Far-field conditions. The disturbance potential and velocity tend to zero at a large distance from the wing:

$$
\varphi(x, z, t) \longrightarrow 0, \quad \nabla \varphi(x, z, t) \longrightarrow 0, \quad \text { as }|x| \longrightarrow \infty \text { or } z \longrightarrow \infty .
$$

Now we present a functional-analytic reformulation of problem (2.2)-(2.6) that will be used in the rest of the paper. We will consider the boundary value problem (2.2)-(2.6) in $L_{p}(-\infty, \infty), 1<p<2$, assuming that the initial conditions are trivial, that is, $\varphi(x, z, 0)=$ $\varphi_{t}(x, z, 0)=0$. It will be clear from the analysis below that it is essential to use $p \neq 2$.

Now we describe the function space for our future solution. We assume that the function $\varphi(x, z, t)$ is absolutely continuous with absolutely continuous first derivatives with respect to the variables $z$ and $t$. Regarding the properties of $\varphi$ as a function of $x$, we make the following assumption. Let $D_{x}$ be a closed linear operator in $L_{p}(-\infty, \infty)$ corresponding to the partial derivative $\partial / \partial x$. Then, we require that

$$
\varphi(\cdot, z, t) \in \Phi\left(D_{x}^{2}\right),
$$

with $\Phi$ being the domain of $D_{x}^{2}$, and $D_{x} \varphi(\cdot, z, t)$ being absolutely continuous with respect to $t$.

We require that (2.2) be satisfied in the sense that

$$
\frac{\partial^{2}}{\partial t^{2}} \varphi(\cdot, z, t)+2 M a_{\infty} \frac{\partial}{\partial t} D_{x} \varphi(\cdot, z, t)=\left(a_{\infty}^{2}\left(1-M^{2}\right) D_{x}^{2}+a_{\infty}^{2} \frac{\partial^{2}}{\partial z^{2}}\right) \varphi(\cdot, z, t) .
$$

The flow tangency condition (2.3) will be understood in the sense that

$$
\int_{-b}^{b}\left|\frac{\partial}{\partial z} \varphi(x, z, t)-w_{a}(x, t)\right|^{p} d x \longrightarrow 0 \quad \text { as } z \longrightarrow 0 .
$$

The Kutta-Joukowski conditions (2.5) will be written in integral form as well. We note that due to our assumptions on $\varphi$, the acceleration potential is well defined. Conditions (2.5) will be replaced with the following requirements:

$$
\begin{gathered}
\int_{-\infty}^{\infty}|\psi(x, z, t)-\psi(x, 0, t)|^{p} d x \longrightarrow 0 \text { as } z \longrightarrow 0, \\
\int_{-\infty}^{-b}|\psi(x, 0, t)|^{p} d x+\int_{b}^{\infty}|\psi(x, 0, t)|^{p} d x=0 .
\end{gathered}
$$

Regarding the initial conditions, we require

$$
\|\varphi(\cdot, z, t)\|_{L^{p}(-\infty, \infty)} \longrightarrow 0, \quad\left\|\frac{\partial}{\partial t} \varphi(\cdot, z, t)\right\|_{L^{p}(-\infty, \infty)} \longrightarrow 0 \quad \text { as } t \longrightarrow 0,0<z<\infty .
$$

In the next sections, we reduce the initial boundary value problem (2.8)-(2.11) to a specific singular integral equation, the Possio integral equation. This derivation is quite nontrivial. 


\section{Modification of (2.8) using Fourier and Laplace transformations}

Let $f(\cdot, z, t) \in L_{p}(-\infty, \infty), z>0, t>0$, be a function that has the following property:

$$
\int_{0}^{\infty}\|f(\cdot, z, t)\|_{L_{p}(-\infty, \infty)} e^{-\sigma t} d t<\infty \quad \text { for } \sigma \geqslant \sigma_{a}>0
$$

In the future, we will omit the subindex $L_{p}(-\infty, \infty)$ using the notation $\|\cdot\|$ for the $L_{p}$ norm. Due to (3.1), we can define the Laplace transform of $f$ with respect to the time variable. We denote the Laplace transform of a function $f$ by the same letter capitalized $(F)$. So, if $\varphi(\cdot, z, t)$ belongs to the class of functions satisfying (3.1), then the Laplace transform of $\varphi(\cdot, z, t)$ is

$$
\Phi(\cdot, z, \lambda)=\int_{0}^{\infty} \varphi(\cdot, z, t) e^{-\lambda t} d t, \quad \Re \lambda \geqslant \sigma_{a}>0,0<z<\infty .
$$

It is clear that from $(2.8)$ for $\varphi(\cdot, z, t)$ we obtain a new equation for $\Phi(\cdot, z, \lambda)$ :

$$
\lambda^{2} \Phi(\cdot, z, \lambda)+2 M a_{\infty} \lambda D_{x} \Phi(\cdot, z, \lambda)=a_{\infty}^{2}\left(1-M^{2}\right) D_{x}^{2} \Phi(\cdot, z, \lambda)+a_{\infty}^{2} \Phi_{z z}(\cdot, z, \lambda) .
$$

In the next step, we apply the Fourier transformation with respect to the variable $x$ to (3.3), and have ("overhat" means the result of the Fourier transformation)

$$
\widehat{\Phi}(\omega, z, \lambda)=\int_{-\infty}^{\infty} \Phi(x, z, \lambda) e^{-i \omega x} d x, \quad-\infty<\omega<\infty .
$$

As it is well known, the Fourier transformation is a bounded linear operator from $L_{p}(-\infty, \infty)$ into $L_{q}(-\infty, \infty)\left(p^{-1}+q^{-1}=1\right), 1<p<2$. Applying the spatial Fourier transformation to (3.3), we obtain a new equation for $\widehat{\Phi}$ :

$$
\left(\lambda^{2}+2 i \omega \lambda M a_{\infty}\right) \widehat{\Phi}(\omega, z, \lambda)=-a_{\infty}^{2}\left(1-M^{2}\right) \omega^{2} \widehat{\Phi}(\omega, z, \lambda)+a_{\infty}^{2} \widehat{\Phi}_{z z}(\omega, z, \lambda) .
$$

Rearranging terms in this equation, we obtain

$$
\widehat{\Phi}_{z z}(\omega, z, \lambda)=\left(1-M^{2}\right) \omega^{2} \widehat{\Phi}(\omega, z, \lambda)+\left(\frac{\lambda^{2}}{a_{\infty}^{2}}+\frac{2 i \omega \lambda M}{a_{\infty}}\right) \widehat{\Phi}(\omega, z, \lambda)
$$

Recalling that $U / a_{\infty}=M$ and denoting $\tilde{\mathcal{\lambda}}=\mathcal{\lambda} / U$, we rewrite (3.6) as

$$
\widehat{\Phi}_{z z}(\omega, z, \lambda)=\left(\tilde{\lambda}^{2} M^{2}+2 i \omega \tilde{\lambda} M^{2}+\omega^{2}\left(1-M^{2}\right)\right) \widehat{\Phi}(\omega, z, \lambda) .
$$

The quadratic polynomial $\left(\widetilde{\lambda}^{2} M^{2}+2 i \omega \tilde{\lambda} M^{2}+\omega^{2}\left(1-M^{2}\right)\right)$ will play an important role in the sequel. We make the following agreement: let us keep the notation $\lambda$ for $\tilde{\lambda}$ and use the notation $\lambda^{\prime}$ for the original $\lambda$; that is, with this agreement, (3.7) obtains the following form:

$$
\widehat{\Phi}_{z z}\left(\omega, z, \lambda^{\prime}\right)=\left(\lambda^{2} M^{2}+2 i \omega \lambda M^{2}+\omega^{2}\left(1-M^{2}\right)\right) \widehat{\Phi}\left(\omega, z, \lambda^{\prime}\right), \quad \lambda^{\prime}=\lambda U .
$$

Let

$$
\Phi(\omega, \lambda)=\lambda^{2} M^{2}+2 i \omega \lambda M^{2}+\omega^{2}\left(1-M^{2}\right)
$$


Let us show that $|\Phi(\omega, \lambda)|$ is bounded below when $\Re \lambda \geqslant \sigma_{a}>0$. Indeed,

$$
|\Phi(\omega, \lambda)|=\left|M^{2}(i \omega+\lambda)^{2}+\omega^{2}\right| \geqslant M^{2}|\lambda+i \omega|^{2} \geqslant M^{2}|\Re \lambda|^{2} \geqslant \frac{M^{2} \sigma_{a}^{2}}{U^{2}}>0 .
$$

From this estimate, it follows that we can define the "positive" square root that we denote by $\sqrt{\Phi(\omega, \lambda)}$ such that

$$
\Re \sqrt{\Phi(\omega, \lambda)}>0 .
$$

Thus, from (3.11) it follows that the differential equation (3.8) has a unique solution satisfying the Far-field conditions, and this solution is

$$
\widehat{\Phi}\left(\omega, z, \lambda^{\prime}\right)=\widehat{\Phi}\left(\omega, 0, \lambda^{\prime}\right) \exp (-z \sqrt{\Phi(\omega, \lambda)}) .
$$

Now we have to satisfy the Kutta-Joukowski conditions and the flow tangency condition.

To write the flow tangency condition, we need a derivative with respect to $z$. Let

$$
\widehat{v}\left(\omega, z, \lambda^{\prime}\right)=\frac{d}{d z} \widehat{\Phi}\left(\omega, z, \lambda^{\prime}\right)=-\sqrt{\Phi(\omega, \lambda)} \widehat{\Phi}\left(\omega, z, \lambda^{\prime}\right) .
$$

In terms of $\widehat{v},(3.12)$ can be written as

$$
\widehat{\Phi}\left(\omega, z, \lambda^{\prime}\right)=-\frac{\widehat{v}\left(\omega, 0, \lambda^{\prime}\right)}{\sqrt{\Phi(\omega, \lambda)}} \exp (-z \sqrt{\Phi(\omega, \lambda)}) .
$$

We notice that the Laplace transform of the acceleration potential is

$$
\Psi\left(\omega, z, \lambda^{\prime}\right)=\lambda^{\prime} \Phi\left(x, z, \lambda^{\prime}\right)+U \Phi\left(x, z, \lambda^{\prime}\right)
$$

and, therefore, using (3.14), we obtain

$$
\widehat{\Psi}\left(\omega, z, \lambda^{\prime}\right)=\left(\lambda^{\prime}+i \omega U\right) \widehat{\Phi}\left(\omega, z, \lambda^{\prime}\right)=-\frac{\left(\lambda^{\prime}+i \omega U\right) \widehat{v}\left(\omega, 0, \lambda^{\prime}\right)}{\sqrt{\Phi(\omega, \lambda)}} \exp (-z \sqrt{\Phi(\omega, \lambda)}) .
$$

From (3.16), we obtain that

$$
\widehat{\Psi}\left(\omega, 0, \lambda^{\prime}\right)=-\frac{\left(\lambda^{\prime}+i \omega U\right) \widehat{v}\left(\omega, 0, \lambda^{\prime}\right)}{\sqrt{\Phi(\omega, \lambda)}} .
$$

At this point, we introduce the standard notation, that is,

$$
\mathcal{A}(x, t)=-\frac{2}{U} \psi(x, 0, t)
$$

We will denote the Laplace transform of $\mathcal{A}$ with respect to the time variable by $A$. Multiplying (3.17) by $2 / U$, we have

$$
\widehat{A}\left(\omega, \lambda^{\prime}\right)=\frac{2(\lambda+i \omega)}{\sqrt{\Phi(\omega, \lambda)}} \widehat{v}\left(\omega, 0, \lambda^{\prime}\right)
$$


or in another form

$$
\widehat{v}\left(\omega, 0, \lambda^{\prime}\right)=\frac{\sqrt{\Phi(\omega, \lambda)}}{2(\lambda+i \omega)} \widehat{A}\left(\omega, \lambda^{\prime}\right),
$$

with $\widehat{v}$ being defined in (3.13).

We notice that if we know $\widehat{A}\left(\omega, \lambda^{\prime}\right)$, then we know the velocity potential, that is,

$$
\widehat{\Phi}\left(\omega, z, \lambda^{\prime}\right)=-\frac{1}{2(\lambda+i \omega)} \exp (-z \sqrt{\Phi(\omega, \lambda)}) \widehat{A}\left(\omega, \lambda^{\prime}\right)
$$

Finally, we obtain the following problem for $\widehat{A}$ :

$$
\begin{aligned}
\widehat{v}\left(\omega, 0, \lambda^{\prime}\right) & =\frac{\sqrt{\Phi(\omega, \lambda)}}{2(\lambda+i \omega)} \widehat{A}\left(\omega, \lambda^{\prime}\right), \\
v\left(x, 0, \lambda^{\prime}\right) & =W_{a}\left(x, \lambda^{\prime}\right), \quad|x|<b, \\
A\left(x, \lambda^{\prime}\right) & =0, \quad|x|>b .
\end{aligned}
$$

System (3.22) is a nonstandard boundary value problem. Indeed, the right-hand side of the first equation in (3.22) has a product of the two functions and, therefore, this product corresponds to a convolution of the function of $x$ corresponding to $\sqrt{\Phi(\omega, \lambda)} / 2(\lambda+i \omega)$ and $\mathcal{A}\left(x, \lambda^{\prime}\right)$. On the other hand, if we restrict $x$ to the interval $[-b, b]$, we know the left-hand side in $x$ representation (it is $\left.W_{a}\left(x, \lambda^{\prime}\right)\right)$. Therefore, the first equation from (3.22) is indeed an integral equation for $\mathcal{A}\left(x, \lambda^{\prime}\right)$ provided that we can restore a function corresponding to the multiple $\sqrt{\Phi\left(\omega, \lambda^{\prime}\right)} / 2(\lambda+i \omega)$. In the next section, we obtain the first main result of the paper. We present a careful derivation of the function of $x$ whose Fourier transformation is given by the aforementioned multiple. This result yields the Possio integral equation (see Theorem 5.13), which will be derived in Section 5 .

\section{Main technical result for reconstruction of the inverse Fourier transform of (3.20)}

The main purpose of this section is representing the right-hand side of (3.20) in the form of a function of $x$ depending parametrically on $\lambda$. As a consequence, incorporating second and third conditions from (3.22) we immediately obtain the desired integral equation. Let

$$
\mathbb{D}(\omega, \lambda)=\frac{\sqrt{\Phi(\omega, \lambda)}}{\lambda+i \omega} .
$$

By direct calculations we obtain

$$
\mathbb{D}(\omega, \lambda)=\frac{|\omega| \sqrt{1-M^{2}}}{\lambda+i \omega} \sqrt{1+\frac{\lambda M^{2}}{1-M^{2}} \frac{\lambda+2 i \omega}{\omega^{2}}} \equiv \frac{|\omega|}{i \omega} \sqrt{1-M^{2}}(1+Q(\omega, \lambda)),
$$

where

$$
Q(\omega, \lambda)=\frac{i \omega}{\lambda+i \omega} \sqrt{1+\frac{\lambda M^{2}}{1-M^{2}} \frac{\lambda+2 i \omega}{\omega^{2}}}-1 .
$$

The following result is valid for the function $Q(\omega, \lambda)$. 
Theorem 4.1. $Q(\cdot, \lambda)$ is a Fourier transform of a function $\mathbf{q} \in L_{p}(-\infty, \infty), p>1$, given explicitly by the following formulas:

$$
\mathbf{q}(x, \lambda)= \begin{cases}-\lambda \frac{e^{-\lambda x}}{\sqrt{1-M^{2}}}-\frac{\lambda}{\pi} \int_{0}^{\alpha_{1}} e^{-\lambda s x} a(x) d s, & x>0, \\ \frac{\lambda}{\pi} \int_{0}^{\alpha_{2}} e^{-\lambda|x| s} a(-s) d s, & x<0,\end{cases}
$$

where

$$
\alpha_{1}=\frac{M}{1+M}, \quad \alpha_{2}=\frac{M}{1-M}, \quad a(s)=\frac{\sqrt{\left(\alpha_{1}-s\right)\left(\alpha_{2}+s\right)}}{1-s}, \quad-\alpha_{2}<s<\alpha_{2} .
$$

Proof. To prove (4.4), we calculate Fourier transform of $\mathbf{q}$ directly and show that it is equal to $\mathcal{Q}(\cdot, \lambda)$ given in $(4.3)$. So, for the Fourier transform, denoted by $\mathbf{F}[\mathbf{q}]$, we have

$$
\begin{aligned}
\mathbf{F}[\mathbf{q}] \equiv & \int_{-\infty}^{\infty} e^{-i \omega x} q(x, \lambda) d x \\
= & \frac{\lambda}{\pi} \int_{-\infty}^{0} e^{-i \omega x} d x \int_{0}^{\alpha_{2}} e^{-\lambda|x| s} a(-s) d s-\frac{\lambda}{1-M^{2}} \int_{0}^{\infty} e^{-\lambda x} e^{-i \omega x} d x \\
& -\frac{\lambda}{\pi} \int_{0}^{\infty} e^{-i \omega x} d x \int_{0}^{\alpha_{1}} e^{-\lambda s x} a(s) d s=I_{1}+I_{2}+I_{3} .
\end{aligned}
$$

Taking into account that $\Re \lambda \geqslant \sigma_{a} \gg 1$, we evaluate $I_{2}$ and have

$$
I_{2}=-\frac{\lambda}{\sqrt{1-M^{2}}} \int_{0}^{\infty} e^{-(\lambda+i \omega) x} d x=-\frac{\lambda}{\sqrt{1-M^{2}}(\lambda+i \omega)} .
$$

Using (4.5), we evaluate $I_{3}$ and have

$$
I_{3}=-\frac{\lambda}{\pi} \int_{0}^{\alpha_{1}} a(s) d s \int_{0}^{\infty} e^{-(\lambda s+i \omega) x} d x=-\frac{\lambda}{\pi} \int_{0}^{\alpha_{1}} \frac{\sqrt{\left(\alpha_{1}-s\right)\left(\alpha_{2}+s\right)}}{(1-s)(i \omega+\lambda x)} d s .
$$

Evaluating $I_{1}$, we obtain

$$
I_{1}=\frac{\lambda}{\pi} \int_{0}^{\alpha_{2}} a(-s) d s \int_{0}^{\infty} e^{(i \omega-\lambda s) x} d x=-\frac{\lambda}{\pi} \int_{0}^{\alpha_{2}} a(-s) \frac{d s}{i \omega-\lambda s} .
$$

Combining together $I_{1}$ and $I_{3}$, we obtain

$$
I_{1}+I_{3}=-\frac{\lambda}{\pi} \int_{-\alpha_{2}}^{\alpha_{1}} \frac{a(s) d s}{i \omega+\lambda s} .
$$

To modify (4.10), let us introduce a new variable of integration:

$$
\xi=s+\alpha_{1} \alpha_{2}=s+M\left(\frac{M}{\left(1-M^{2}\right)}\right)=s+M \beta, \quad \beta=\frac{M}{\left(1-M^{2}\right)} .
$$


The following relations can be easily verified:

$$
\begin{gathered}
\alpha_{1}-s=\beta-\xi, \quad \alpha_{2}+s=\frac{M}{1-M}+\xi-\frac{M^{2}}{1-M^{2}}=\beta+\xi ; \\
1-s=\xi+\frac{1}{1-M^{2}}, \quad \frac{i \omega}{\lambda}+s=\frac{i \omega}{\lambda}+\xi-M \beta .
\end{gathered}
$$

Using (4.12) and setting $z=(i \omega / \lambda)$, we rewrite $(4.10)$ as

$$
I_{1}+I_{3}=-\frac{1}{\pi} \int_{-\beta}^{\beta} \frac{\sqrt{(\beta-\xi)(\beta+\xi)} d \xi}{(1-\xi+M \beta)((i \omega / \lambda)+\xi-M \beta)}=-\frac{1}{\pi} \int_{-\beta}^{\beta} \frac{\sqrt{\beta^{2}-\xi^{2}} d \xi}{(1-\xi+M \beta)(z+\xi-M \beta)}
$$

Making transformation $\xi \mapsto-\xi$ in (4.13) and taking into account that $(1 / \beta)+M=1 / M$, we get

$$
\begin{aligned}
I_{1}+I_{3} & =-\frac{1}{\pi} \int_{-\beta}^{\beta} \frac{\sqrt{\beta^{2}-\xi^{2}} d \xi}{(1+\xi+M \beta)(z-\xi-M \beta)} \\
& =\frac{1}{\pi} \int_{-\beta}^{\beta} \frac{\sqrt{1-(\xi / \beta)^{2}} d \xi}{((1 / \beta)+M+(\xi / \beta))((\xi / \beta)+M-(z / \beta)) \beta} \\
& =\frac{1}{\pi} \int_{-\beta}^{\beta} \frac{\sqrt{1-(\xi / \beta)^{2}} d \xi}{\beta((1 / M)+(\xi / \beta))((\xi / \beta)+M-(z / \beta))} .
\end{aligned}
$$

Let $\eta=\xi / \beta$, then

$$
I_{1}+I_{3}=\frac{1}{\pi} \int_{-1}^{1} \frac{\sqrt{1-\eta^{2}} d \eta}{(\eta+(1 / M))(\eta+M-(z / \beta))}=\frac{\beta}{\pi(1+z)}\left(i_{1}-i_{2}\right),
$$

where

$$
i_{1}=\int_{-1}^{1} \frac{\sqrt{1-\eta^{2}} d \eta}{(\eta+M-(z / \beta))}, \quad i_{2}=\int_{-1}^{1} \frac{\sqrt{1-\eta^{2}} d \eta}{\eta+(1 / M)}
$$

To evaluate the second integral of (4.16), we need the statement below, which will be proved after the proof of Theorem 4.1 .

Lemma 4.2. The following formula holds for $|a|>1$ :

$$
\int_{-1}^{1} \frac{\sqrt{1-x^{2}}}{x+a} d x=\pi\left(a-\operatorname{sign}(\Re a) \sqrt{a^{2}-1}\right) .
$$

To evaluate $i_{1}$ from (4.16), we can derive a formula similar to (4.17). However, we notice that the integrand of $i_{1}$ is of the form $\sqrt{1-\eta^{2}} /(\eta+\varkappa)$, where $\varkappa$ is a complex number with 
a nonvanishing imaginary part (except for the one point where $\varkappa=M<1$ ). For such $\varkappa$, one can reconstruct the proof of the formula similar to (4.17) and have

$$
i_{1}=\pi\left(M-\frac{z}{\beta}+\sqrt{\left(M-\frac{z}{\beta}\right)^{2}-1}\right) .
$$

Substituting $1 / M$ for $a$ in (4.17), we obtain

$$
\begin{aligned}
\frac{i_{1}-i_{2}}{\pi} & =M-\frac{z}{\beta} \\
& =\sqrt{\left(M-\frac{z}{\beta}\right)^{2}-1}-\frac{1}{M}-\sqrt{\frac{1}{M^{2}}-1} \\
& =-\frac{1+z}{\beta}+\frac{\sqrt{1-M^{2}}}{M}+\frac{1}{\beta} \sqrt{z^{2}-\beta^{2}\left(1-M^{2}\right)-2 z M \beta} \\
& =\frac{1+z}{\beta}\left[-1+\frac{1}{1+z} \frac{1}{\sqrt{1-M^{2}}}+\frac{\sqrt{z^{2}-2 z \gamma-\gamma}}{1+z}\right],
\end{aligned}
$$

where $\gamma=\beta M$ and $\beta$ is defined in (4.11).

Returning to (4.15), we obtain

$$
I_{1}+I_{3}=-1+\frac{1}{(1+z) \sqrt{1-M^{2}}}+\frac{1}{1+z} \sqrt{z^{2}-2 z \gamma-\gamma}
$$

Now we can finalize the expression for $Q$ combining (4.7) with (4.20) to have

$$
Q(x, \lambda)=-\frac{\lambda}{\sqrt{1-M^{2}}(\lambda+i \omega)}+\frac{1}{(1+z) \sqrt{1-M^{2}}}+\frac{z}{1+z} \sqrt{1-\frac{2 \gamma}{z}-\frac{\gamma}{z^{2}}}-1 .
$$

Recalling that $z=i \omega / \lambda$ and noticing that $(2 \gamma / z)+\left(\gamma / z^{2}\right)=(2 M \beta \lambda / i \omega)-\left(M \beta \lambda^{2} / \omega^{2}\right)$, we obtain from (4.21) that

$$
\begin{aligned}
Q(x, \lambda) & =\frac{i \omega}{\lambda+i \omega} \sqrt{1-\frac{2 M \beta \lambda}{i \omega}+\frac{M \beta \lambda^{2}}{\omega^{2}}}-1 \\
& =\frac{i \omega}{\lambda+i \omega} \sqrt{1+\frac{2 M \beta \lambda i \omega+M \beta \lambda^{2}}{\omega^{2}}}-1 \\
& =\frac{i \omega}{\lambda+i \omega} \sqrt{1+\frac{\lambda M \beta}{\omega^{2}}(\lambda+2 i \omega)}-1
\end{aligned}
$$

This formula coincides with (4.3). To complete the proof of the theorem, it remains to show that $\mathbf{q} \in L_{p}(-\infty, \infty)$. As the first observation, we notice that it suffices to prove the result for the case $\lambda=1$. Indeed, from (4.4) we obtain

$$
|\mathbf{q}(x, \lambda)| \leqslant|\lambda||q(x, \sigma)|, \quad \sigma=\Re \lambda \geqslant \sigma_{a}>0 .
$$


Thus,

$$
\int_{-\infty}^{\infty}|\mathbf{q}(x, \lambda)|^{p} d x \leqslant|\lambda|^{p} \int_{-\infty}^{\infty}|\mathbf{q}(x, \sigma)|^{p} d x=|\lambda|^{p} \int_{-\infty}^{\infty}|\mathbf{q}(\sigma x, 1)|^{p} d x=\frac{|\lambda|^{p}}{\sigma} \int_{-\infty}^{\infty}|\mathbf{q}(\xi, 1)|^{p} d \xi .
$$

Taking into account $|a(s)| \leqslant C<\infty$ for $-\alpha_{2}<s<\alpha_{1}$, we get the following estimates:

$$
\begin{gathered}
\left|\int_{0}^{\alpha_{1}} e^{-x s} a(s) d s\right| \leqslant C \frac{1-e^{-\alpha_{1} x}}{1+x}, \quad x>0, \\
\left|\int_{0}^{\alpha_{2}} e^{-|x| s} a(-s) d s\right| \leqslant C \frac{1-e^{-\alpha|x|}}{1+|x|}, \quad x<0 .
\end{gathered}
$$

From estimates (4.25) we obtain

$$
\int_{-\infty}^{\infty}|q(x, 1)|^{p} d x \leqslant C\left[\int_{-\infty}^{0}\left(\frac{1-e^{-\alpha_{2}|x|}}{1+|x|}\right)^{p} d x+\int_{0}^{\infty}\left(\frac{1-e^{-\alpha_{1} x}}{1+|x|}\right)^{p} d x\right]<\infty \quad \text { for } p>1 .
$$

This estimate means that $\mathbf{q} \in L_{p}(-\infty, \infty)$.

The theorem is completely shown.

Now we complete the proof of the lemma.

Proof of Lemma 4.2. We consider the following integral:

$$
I=\int_{-1}^{1} \frac{\sqrt{1-x^{2}}}{x+a} d x, \quad|a|>1
$$

Let $x=\cos \theta$, then

$$
I=\int_{0}^{\pi} \frac{\sin ^{2} \theta}{\cos \theta+a} d \theta=\frac{1}{2}\left[\int_{0}^{\pi} \frac{d \theta}{\cos \theta+a}-\int_{0}^{\pi} \frac{\cos 2 \theta}{\cos \theta+a} d \theta\right] \equiv \frac{1}{2}\left(j_{1}-j_{2}\right) .
$$

Evaluating $j_{1}$, we have

$$
j_{1}=\int_{-\pi}^{\pi} \frac{d \theta}{e^{i \theta}+e^{-i \theta}+2 a} .
$$

Integrating in the complex plane, we assume that $z=e^{i \theta}$, and thus linear integral can be represented as a contour integral along a unit circle centered at the origin:

$$
j_{1}=\frac{1}{i} \oint_{C_{1}(0)} \frac{d z}{z\left(z+z^{-1}+2 a\right)}=\frac{1}{i} \oint_{C_{1}(0)} \frac{d z}{z^{2}+2 z a+1} .
$$

The roots of the denominator are $z_{1}=-a+\sqrt{a^{2}-1}, z_{2}=z_{1}^{-1}=-a-\sqrt{a^{2}-1}$. First, we show that for $|a|>1$, neither of the roots lie on the circumference. Indeed, assume that $\left|z_{1}\right|=1$, then $\left|z_{2}\right|=1$. Therefore,

$$
z_{1}=-a+\sqrt{a^{2}-1}=e^{i \psi}, \quad z_{2}=-a-\sqrt{a^{2}-1}=e^{-i \psi} .
$$


From (4.31), it follows that $a=-\cos \psi$ which contradicts our assumption, $|a|>1$. Thus, one of the roots is inside the circle $C_{1}(0)=\{z:|z|=1\}$ and the second one is outside. Let $\left|z_{1}\right|=$ $\left|-a+\sqrt{a^{2}-1}\right|<1$, then by the residue theorem we have

$$
j_{1}=\frac{1}{i} \oint \frac{d z}{\left(z-z_{1}\right)\left(z-z_{2}\right)}=\frac{\pi}{\sqrt{a^{2}-1}} .
$$

Finally, we evaluate $j_{2}$ from (4.28):

$$
\begin{aligned}
j_{2} & =\frac{1}{2} \int_{-\pi}^{\pi} \frac{\cos 2 \theta}{\cos \theta+a} d \theta=\frac{1}{2} \int_{-\pi}^{\pi} \frac{e^{2 i \theta}+e^{-2 i \theta}}{e^{i \theta}+e^{-i \theta}+a} d \theta \\
& =\frac{1}{2 i} \oint_{C_{1}(0)} \frac{\left(z^{2}+z^{-} 2\right) d z}{z^{2}+2 a z+1}=\frac{1}{2 i} \oint_{C_{1}(0)} \frac{d z\left(z^{4}+1\right)}{z^{2}\left(z-z_{1}\right)\left(z-z_{2}\right)} .
\end{aligned}
$$

We have to evaluate the residues at the points $z=0$ and $z \equiv z_{1}=-a+\sqrt{a^{2}-1}$. We have

(a) $\operatorname{Res}[f ; 0]=\left.\pi\left(\frac{z^{4}+1}{z^{2}+2 a z+1}\right)^{\prime}\right|_{z=0}=-2 \pi a$,

(b) $\operatorname{Res}\left[f ; z_{1}\right]=\left.\pi \frac{z^{2}+z^{-2}}{2 \sqrt{z^{2}-1}}\right|_{z=-a+\sqrt{a^{2}-1}}=\pi \frac{\left(a-\sqrt{a^{2}-1}\right)^{2}+\left(a+\sqrt{a^{2}-1}\right)^{2}}{2 \sqrt{a^{2}-1}}=\pi \frac{2 a^{2}-1}{\sqrt{a^{2}-1}}$.

Using (4.34), we get

$$
j_{2}=\operatorname{Res}[f ; 0]+\operatorname{Res}\left[f ; z_{1}\right]=\frac{\pi}{\sqrt{a^{2}-1}}\left(2 a^{2}-1-2 a \sqrt{a^{2}-1}\right)=\frac{\pi}{\sqrt{a^{2}-1}}\left(a-\left(\sqrt{a^{2}-1}\right)\right)^{2},
$$

which together with (4.32) yields

$$
j_{1}-j_{2}=\frac{\pi}{\sqrt{a^{2}-1}}\left(1-\left(a-\sqrt{a^{2}-1}\right)^{2}\right)=\frac{\pi}{\sqrt{a^{2}-1}}\left(1-a^{2}+a \sqrt{a^{2}-1}\right)=2 \pi\left(a-\sqrt{a^{2}-1}\right) .
$$

Inserting (4.36) into (4.28), we obtain (4.17).

The lemma is completely shown.

Remark 4.3. Now we outline how the results obtained in this section will be used for rewriting the first equation of (3.22) in the form of an integral equation with respect to the unknown function $A\left(\cdot, \lambda^{\prime}\right)$. If we consider that integral equation for $|x|<b$, then the left-hand side will be given explicitly as the Laplace transform of the given downwash function $W_{a}\left(\cdot, \lambda^{\prime}\right)$. The right-hand side will be the convolution of the inverse Fourier transforms of the functions from the right-hand side of (3.22). In other words, the right-hand side will be given as an integral convolution operation over unknown function $A\left(\cdot, \lambda^{\prime}\right)$. 
To achieve our goal, we will use (4.4), which gives an explicit function $\mathbf{q}$ whose Fourier transform is (4.3). It turns out that (4.3) is not a convenient formula to work with. Namely, using (4.4), we obtain the following Fourier representation for $Q$ :

$$
Q(\omega, \lambda)=-\frac{\lambda}{\sqrt{1-M^{2}}} \frac{1}{\lambda+i \omega}-\lambda \int_{0}^{\alpha_{1}} \frac{a(s)}{\lambda s+i \omega} d s+\lambda \int_{0}^{\alpha_{2}} \frac{a(-s)}{\lambda-i \omega} d s, \quad \Re \lambda \geqslant \delta_{a}>0 .
$$

In terms of (4.37), we obtain that our main multiplier $\mathbb{D}(\omega, \lambda)$ (from $(4.2)$ ) can be represented in the form

$$
\begin{aligned}
\frac{1}{\sqrt{1-M^{2}}} \mathbb{D}(\omega, \lambda)= & \frac{|\omega|}{i \omega}(1+Q(\omega, \lambda)) \\
= & \frac{|\omega|}{i \omega}-\frac{|\omega|}{i \omega} \frac{1}{\sqrt{1-M^{2}}} \frac{\lambda}{\lambda+i \omega}-\frac{|\omega|}{i \omega} \lambda \int_{0}^{\alpha_{1}} \frac{a(s) d s}{\lambda s+i \omega} \\
& +\frac{|\omega|}{i \omega} \lambda \int_{0}^{\alpha_{2}} \frac{a(-s) d s}{\lambda s-i \omega}, \quad \Re \lambda \geqslant \delta_{a}>0 .
\end{aligned}
$$

In the next section, we prove that each function at the right-hand side of (4.38) is the socalled Mikhlin multiplier. Evidently each individual multiplier looks simpler than $\mathbb{D}(\cdot, \lambda)$, which allows us to construct an operator in $L_{p}(-\infty, \infty)$ which corresponds to the entire multiplier $\mathbb{D}$. In particular, we derive the formulas for the operators corresponding to all the multipliers of (4.38) and then sum them up.

\section{Mikhlin multipliers}

In this section, we derive the desired form of the Possio equation as an integral equation, and show that the integral operator in this equation is bounded in $L_{p}(-\infty, \infty), 1<p<2$. The main tool in this derivation is the notion of Mikhlin multipliers [24].

Definition 5.1. Let $g$ and $f$ be two functions from $L_{p}(-\infty, \infty), p>1$, and let $G$ and $F$ be their Fourier transforms. Let there exist a function $\mu$ that relates $G$ and $F$ by the rule

$$
G(\omega)=\mu(\omega) F(\omega) .
$$

If $\mu$ is a continuously differentiable function (with one possible exception at $\omega=0$ ) such that

$$
|\mu(\omega)|+|\omega|\left|\mu^{\prime}(\omega)\right| \leqslant C<\infty,
$$

then $\mu$ is called a Mikhlin multiplier.

Proposition 5.2 (see [24] for the proof). Let $f$ and $g$ be the functions from Definition 5.1, and let $\mu$ be a Mikhlin multiplier. Then, there exists a bounded linear operator $\mathbb{Q}$ in $L_{p}(-\infty, \infty)$ that relates $f$ and $g$ by the rule

$$
g=\mathbb{Q}[f] .
$$

The norm of the operator $\mathbb{Q}$ can be estimated as follows:

$$
\|\mathbb{Q}\|_{L_{p}(-\infty, \infty)} \leqslant C M_{p}
$$

where $C$ is a constant from (5.2), and $M_{p}$ is a constant depending only on $p$. 
Our first result is related to the function from (3.20), that is, $\sqrt{\Phi(\omega, \lambda)} / 2(\lambda+i \omega)$. As pointed out, if we can identify a function from $L_{p}(-\infty, \infty)$ whose Fourier transform coincides with the right-hand side of (3.20), then we will be able to rewrite (3.20) in a standard form, that is, as an integral convolution equation. Our first statement is the following lemma.

Lemma 5.3. The function

$$
\mathbb{D}(\omega, \lambda)=\frac{\sqrt{\Phi(\omega, \lambda)}}{\lambda+i \omega}
$$

is a Mikhlin multiplier.

Proof. Since $\Phi(\omega, \lambda)=M^{2} \lambda^{2}+2 i \omega \lambda M^{2}+\left(1-M^{2}\right) \omega^{2}$, we have

$$
\mathbb{D}^{\prime}(\omega, \lambda)=\frac{i \lambda M^{2}+\omega\left(1-M^{2}\right)}{(\lambda+i \omega) \sqrt{\Phi(\omega, \lambda)}}-\frac{i \sqrt{\Phi(\omega, \lambda)}}{(\lambda+i \omega)^{2}} .
$$

From (5.6), it can be readily seen that

$$
\left|\omega \mathbb{D}^{\prime}(\omega, \lambda)\right| \leqslant C<\infty .
$$

We note that estimate (5.7) is valid for all $\lambda$ including zero (though it is not our case). Indeed, we have $\mathbb{D}^{\prime}(\omega, 0)=\left(\sqrt{1-M^{2}} / i \omega\right)+\left(i \sqrt{1-M^{2}} / \omega\right)=0$. Therefore, the function $\mathbb{D}(\omega, \lambda)$ is a Mikhlin multiplier.

The lemma is shown.

The next statement is related to the function $(\lambda+i \omega)^{-1}$ which is a part of the main multiplier (5.5) (see (4.38)).

Lemma 5.4. (1) The function $(\lambda+i \omega)^{-1}$ is a Mikhlin multiplier. formula:

(2) Let $f \in L_{p}(-\infty, \infty), p>1$, and let $g$ be related to $f$ through the operator $Q_{1}$ by the following

$$
g(x, \lambda)=\left(Q_{1} f\right)(x)=\int_{-\infty}^{x} e^{-\lambda(x-\eta)} f(\eta) d \eta
$$

Then, the Fourier transforms are related through the multiplier

$$
G(\omega, \lambda)=\frac{1}{\lambda+i \omega} F(\omega)
$$

(3) $\mathcal{Q}_{1}$ is a bounded operator in $L_{p}(-\infty, \infty)$ for each $\lambda$ such that $\mathfrak{R} \lambda \geqslant \sigma_{a}$.

Proof. (1) To check that $(\lambda+i \omega)^{-1}$ is a Mikhlin multiplier, it suffices to verify that for $\Re \lambda \geqslant \sigma_{a}>$ 0 , the following estimate holds:

$$
\left|(\lambda+i \omega)^{-1}\right|+\left|\omega(\lambda+i \omega)^{-2}\right| \leqslant C<\infty, \quad|\omega|<\infty,
$$

which is clearly the case. 
(2) Let us evaluate the Fourier transform of both parts of representation (5.8) and have

$$
\begin{aligned}
G(\omega, \lambda) & =\int_{-\infty}^{\infty} e^{i \omega x} d x \int_{-\infty}^{\infty} e^{-\lambda(x-\eta)} f(\eta) d \eta=\int_{-\infty}^{\infty} f(\eta) e^{\lambda \eta} d \eta \int_{\eta}^{\infty} e^{(\lambda+i \omega) x} d x \\
& =\frac{1}{\lambda+i \omega} \int_{-\infty}^{\infty} f(\eta) e^{i \omega \eta} d \eta=\frac{1}{\lambda+i \omega} F(\omega)
\end{aligned}
$$

Equation (5.11) means that if $Q_{1}$ is defined by (5.8), then the corresponding multiplier is $(\lambda+i \omega)^{-1}$.

(3) It remains to prove that $Q_{1}$ is a bounded operator in $L_{p}(-\infty, \infty)$. We have

$$
\begin{aligned}
\left\|Q_{1}[f]\right\|_{L_{p}(-\infty, \infty)} & =\left(\int_{-\infty}^{\infty} d x\left|\int_{-\infty}^{x} f(\eta) e^{-\lambda(x-\eta)} d \eta\right|^{p}\right)^{1 / p} \\
& \leqslant\left(\int_{-\infty}^{\infty} d x\left|\int_{-\infty}^{x}\right| f(\eta)\left|e^{-\sigma(x-\eta)} d \eta\right|^{p}\right)^{1 / p},
\end{aligned}
$$

where $\sigma=\mathfrak{R} \lambda \geqslant \sigma_{a}>0$. Let us use $y$ instead of $\eta$ in (5.12), that is, $y=x-\eta$, and have

$$
\begin{aligned}
\left\|Q_{1}[f]\right\|_{L_{p}(-\infty, \infty)} & \leqslant\left(\int_{-\infty}^{\infty} d x\left|\int_{0}^{\infty} f(x-y) e^{-\sigma y} d y\right|^{p}\right)^{1 / p} \\
& \leqslant \int_{0}^{\infty} e^{-\sigma y} d y\left(\int_{-\infty}^{\infty}|f(x-y)|^{p} d x\right)^{1 / p} \\
& \leqslant \frac{1}{\sigma_{a}}\|f\|_{L_{p}(-\infty, \infty) .} .
\end{aligned}
$$

In the last step, we have used the Minkowski inequality.

The lemma is shown.

In our next statement, we give only the formulation of the result since the proof can be easily reconstructed from the proof of Lemma 5.4.

Lemma 5.5. (1) The function $(\lambda-i \omega)^{-1}$ is a Mikhlin multiplier. formula:

(2) Let $f \in L_{p}(-\infty, \infty), p>1$, and let $g$ be related to $f$ through the operator $Q_{3}$ by the following

$$
g(x, \lambda)=\left(Q_{3} f\right)(x)=\int_{x}^{\infty} e^{-\lambda(\xi-x)} f(\xi) d \xi
$$

Then, the Fourier transforms are related through the multiplier

$$
G(\omega, \lambda)=\frac{1}{\lambda-i \omega} F(\omega)
$$

(3) $Q_{3}$ is a bounded operator in $L_{p}(-\infty, \infty)$ for each $\lambda$ such that $\mathfrak{R} \lambda \geqslant \sigma_{a}$. 
To present our next result, we have to introduce the following notations.

(i) $\varepsilon_{1}(z)$ is "the exponential integral" [25] defined by the formula

$$
\varepsilon_{1}(z)=\frac{1}{\pi} \int_{z}^{\infty} \frac{e^{-\tau}}{\tau} d \tau, \quad \Re z>0 .
$$

The following properties of $\varepsilon_{1}$ will be needed in the sequel. From (5.16), we get

$$
\varepsilon_{1}^{\prime}(z)=-\frac{e^{-z}}{\pi z}=-\frac{1}{\pi z}+\frac{1+e^{-z}}{\pi z}
$$

which yields another representation for $\varepsilon_{1}(z)$ :

$$
\varepsilon_{1}(z)=-\frac{1}{\pi} \ln z+\int_{1}^{z} \frac{1-e^{-\tau}}{\tau} d \tau .
$$

Equation (5.18) means that $\varepsilon_{1}$ is an analytic function correctly defined on the complex plane with the branch cut along the negative real semiaxis including zero. From (5.18), it also follows that $\mathcal{\varepsilon}_{1}$ belongs to $L_{p}(-b, b)$ for any $p \geqslant 1$.

(ii) Let us introduce a new function by the formula

$$
g_{-}(\lambda, x)=e^{-i \lambda x} \varepsilon_{1}(\lambda(b-x)), \quad|x|<b .
$$

For each $x, g_{-}$is an analytic function of $\lambda$ on the complex plane with the branch cut along the negative real semiaxis and

$$
\int_{-b}^{b}\left|g_{-}(\lambda, x)\right|^{p} d x<\infty, \quad p \geqslant 1
$$

Below we define several linear operators that we need in the future.

(iii) Let $D$ be a projection, that is,

$$
(\not P f)(x)=f(x) \quad \text { for }|x|<b, \quad(\not f)(x)=0 \quad \text { for }|x|>b .
$$

(iv) Let $\mathscr{t}$ be the Hilbert transformation defined by [26, 27]

$$
(\mathscr{L} f)(x)=\frac{1}{\pi} \int_{-\infty}^{\infty} \frac{f(\xi)}{x-\xi} d \xi
$$

where " $*$ " (star) means that the integral is understood as a Cauchy principal value integral. Regarding the operator $\mathscr{t}$, we need the following result.

For any $f \in L_{p}(-\infty, \infty)$, (5.22) defines a function $\widetilde{F}=\mathscr{\ell}[f] \in L_{p}(-\infty, \infty)$. In addition,

$$
\mathscr{L}[\mathscr{L}[f]]=-f .
$$

The Mikhlin multiplier, corresponding to the Hilbert transformation, is given explicitly by [26]

$$
\Gamma(\omega)=\frac{|\omega|}{i \omega}, \quad-\infty<\omega<\infty
$$


(v) Let $\mathfrak{L}_{b}$ be a "finite Hilbert transformation" defined by

$$
\left(\mathscr{L}_{b} f\right)(x)=\frac{1}{\pi} \int_{-b}^{b_{*}} \frac{f(\xi)}{x-\xi} d \xi
$$

(vi) Let $Q_{1}$ be a Volterra integral operator defined in (5.8):

$$
\left(Q_{1} f\right)(x)=\int_{-\infty}^{x} e^{-\lambda(x-\xi)} f(\xi) d \xi, \quad f \in L_{p}(-\infty, \infty) .
$$

(vii) Let $\mathcal{L}(\lambda)$ be the following operator:

$$
\mathcal{L}(\lambda) f=\int_{-b}^{x} e^{-\lambda(x-\xi)} f(\xi) d \xi=\left(Q_{1} p\right) f, \quad f \in L_{p}(-\infty, \infty),|x|<b .
$$

(viii) Let $L(\lambda, f)$ be the following linear functional:

$$
L(\lambda, f)=\int_{-b}^{b} e^{\lambda \eta} f(\eta) d \eta
$$

We notice that $L$ defines a bounded linear functional in $L_{p}(-b, b), p \geqslant 1$.

Now we are in a position to present the next result.

Lemma 5.6. The following formula is valid for the linear mapping $D \mathscr{H} Q_{1} D$ in $L_{p}(-\infty, \infty)$ :

$$
\begin{aligned}
& \left(\not \mathscr{H} Q_{1} \not\right)[f]=\left(\mathscr{\ell}_{b} \mathcal{L}(\lambda)\right)[f]-g_{-}(\lambda, x) L(\lambda, f) \quad \text { for }|x|<b \text {, } \\
& \left(\not \mathscr{H} Q_{1} p\right)[f]=0 \text { for }|x|>b \text {. }
\end{aligned}
$$

Proof. Using (5.21), (5.22), and (5.26), we get for $f \in L_{p}(-\infty, \infty)$ and $|x|<b$,

$$
\begin{aligned}
\left(\mathscr{H} Q_{1} D\right)[f] & =\mathscr{H}\left[\int_{-\infty}^{x} e^{-\lambda(x-\xi)}(D f)(\xi) d \xi\right] \\
& =\frac{1}{\pi} \int_{-\infty}^{\infty} \frac{d \xi}{x-\xi} \int_{-b}^{\xi} e^{-\lambda(\xi-\eta)}(D f)(\eta) d \eta \\
& =\frac{1}{\pi}\left[\int_{-\infty}^{-b_{*}}+\int_{-b}^{b_{*}}+\int_{b}^{\infty *}\right] \frac{d \xi}{x-\xi} \int_{-b}^{\xi} e^{-\lambda(\xi-\eta)}(D f)(\eta) d \eta \\
& \equiv i_{1}+i_{2}+i_{3} .
\end{aligned}
$$

For the integral $i_{1}$, we obtain the following result: if $\xi<-b$, then $\eta<\xi<-b$, and thus for such $\eta, p[f](\eta)=0$, which yields $i_{1}=0$.

Using definitions (5.25) and (5.27), we obtain that $i_{2}$ can be given as

$$
i_{2}=\frac{1}{\pi} \int_{-b}^{b_{*}} \frac{d \xi}{x-\xi} \int_{-b}^{\xi} e^{-\lambda(\xi-\eta)} f(\eta) d \eta=\left(\mathscr{\ell}_{b} \mathcal{L}(\lambda) D\right)[f], \quad|x|<b
$$


Finally, we consider $i_{3}$ and have

$$
i_{3}=\frac{1}{\pi} \int_{-b}^{\infty} \frac{e^{-\lambda \xi}}{x-\xi} d \xi \int_{-b}^{b} e^{\lambda \eta} f(\eta) d \eta=\frac{1}{\pi} \int_{-b}^{\infty} \frac{e^{-\lambda \xi}}{x-\xi} d \xi L(\lambda, f), \quad|x|<b,
$$

with $L(\lambda, f)$ being defined as (5.28).

Consider an integral from (5.32) (we are interested only in the case $|x|<b$ ):

$$
\frac{1}{\pi} \int_{b}^{\infty} \frac{e^{-\lambda \xi}}{x-\xi} d \xi=-\frac{1}{\pi} \int_{b-x}^{\infty} \frac{e^{-\lambda(\tau+x)}}{\tau} d \tau=-\frac{e^{-\lambda x}}{\pi} \int_{b-x}^{\infty} \frac{e^{-\lambda \tau}}{\tau} d \tau=-\frac{e^{-\lambda x}}{\pi} \int_{\lambda(b-x)}^{\infty} \frac{e^{-\tau}}{\tau} d \tau=-e^{-\lambda x} \mathcal{\varepsilon}_{1}(\lambda(b-x)) .
$$

In (5.33), we have used definition (5.16). Based on (5.19), we obtain (5.29).

The lemma is completely shown.

Corollary 5.7. The following formula holds for any $f \in L_{p}(-\infty, \infty)$ :

$$
\left(p \mathscr{L C}_{2} D\right)[f]=\int_{0}^{\alpha_{1}} a(s)\left[\left(\mathscr{L}_{b} \mathcal{L}(\lambda s)\right)[f]+g_{-}\left(\lambda_{s}, x\right) L(\lambda s, f)\right] d s, \quad|x|<b,
$$

where $Q_{2}$ is an operator corresponding to a Mikhlin multiplier $\int_{0}^{\alpha_{1}} a(s) d s /(\lambda s+i \omega)$ with $g_{-}, \mathcal{L}(\lambda)$, and $L$ being defined in (5.19), (5.27), and (5.28), respectively.

Proof. First, we notice that both functions $\left(\lambda_{s}+i \omega\right)^{-1}$ and $\left(\lambda_{s}-i \omega\right)^{-1}$ for $-\alpha_{2} \leqslant s \leqslant \alpha_{1}, \mathfrak{R} \lambda \geqslant$ $\sigma_{a}>0$ are the Mikhlin multipliers. We want to identify the operator that corresponds to the multiplier

$$
\varkappa_{1}(\omega)=\int_{0}^{\alpha_{1}} \frac{a(s) d s}{\lambda s+i \omega} \quad\left(\text { or } \varkappa_{2}(\omega)=\int_{0}^{\alpha_{2}} \frac{a(-s) d s}{\lambda s-i \omega}\right)
$$

with $\alpha_{1}$ being defined in (4.5). By Lemma 5.4, the operator corresponding to the multiplier $(\lambda s+i \omega)^{-1}$ can be defined as follows:

$$
\text { if } G(\lambda, s)=\frac{1}{\lambda s+i \omega} F(\lambda), \quad \text { then } g(x, s)=\int_{-\infty}^{x} e^{-\lambda s(x-\xi)} f(\xi) d \xi .
$$

Applying (5.36) to $f \in L_{p}(-b, b)$, multiplying by $a$, and integrating, we obtain

$$
\int_{0}^{\alpha_{1}} g(x, s) a(s) d s=\int_{0}^{\alpha_{1}} a(s) d s \int_{-b}^{x} e^{-\lambda s(x-\xi)} f(\xi) d \xi .
$$

Taking into account that the factor $|\omega|(i \omega)^{-1}$ corresponds to the application of the Hilbert transformation, we get

$$
\mathscr{H}\left[\int_{0}^{\alpha_{1}} g(x, s) a(s) d s\right]=\int_{0}^{\alpha_{1}} a(s) \mathscr{d}\left[\int_{-b}^{x} e^{-\lambda s(x-\xi)} f(\xi) d \xi\right] d s .
$$

In (5.38), we have changed the order of the two integrals; one is a standard integral and another one is "the star" integral. This step is justified in our case since $f \in L_{p}(-b, b)$ and $a$ is a continuous function [27]. Therefore, if we denote by $Q_{2}$ the operator corresponding to the multiplier $\int_{0}^{\alpha_{1}} a(s)\left(d s /\left(\lambda_{s}+i \omega\right)\right)$, the following formula holds:

$$
\left(D \mathscr{H} Q_{2} p\right)[f]=\int_{0}^{\alpha_{1}} a(s)\left[\left(\mathscr{L}_{b} \mathcal{L}(\lambda s)\right)[f]+g_{-}(\lambda s, x) L(\lambda s, f)\right] d s, \quad|x|<b,
$$

with $g_{-}, \mathcal{L}(\lambda)$, and $L$ being defined in (5.19), (5.27), and (5.28), respectively.

The proof of the corollary is complete. 
Lemma 5.8. The following formula is valid for the mapping $D \mathcal{H} Q_{3} D$ in $L_{p}(-\infty, \infty)$ :

$$
\begin{aligned}
\left(\operatorname{D\ell Q}_{3} p\right)[f]= & \left(\mathscr{H}_{b} \mathcal{L}^{*}(\lambda)\right)[f]+g_{+}(\lambda, x) L(-\lambda, f) \text { for }|x|<b, \\
& \left(\operatorname{DHC}_{3} \not\right)[f]=0 \text { for }|x|>b .
\end{aligned}
$$

Here $Q_{3}$ is an operator corresponding to the multiplier $(\lambda-i \omega)^{-1}$ (see Lemma 5.5); a linear operator $\mathcal{L}^{*}$ is defined by

$$
\text { if } \mathcal{L}^{*} f=g, \quad \text { then } g(x)=\int_{x}^{b} e^{-\lambda(\xi-x)} f(\xi) d \xi, \quad|x|<b \text {. }
$$

The function $g_{+}(\lambda, \cdot)$ is defined by the formula

$$
g_{+}(\lambda, x)=e^{\lambda x} \varepsilon_{1}(\lambda(b+x)), \quad|x|<b,
$$

where $\boldsymbol{\varepsilon}_{1}$ is an "exponential integral" from (5.16).

Proof. We discuss only the case $|x|<b$ since the opposite case for $|x|>b$ is obvious. For $f \in$ $L_{p}(-\infty, \infty)$, we have

$$
\begin{aligned}
\left(\mathscr{H} Q_{3} p\right)[f] & =\mathscr{\ell}\left[\int_{x}^{\infty} e^{-\lambda(\xi-x)}(D f)(\xi) d \xi\right] \\
& =\mathscr{H}\left[\int_{x}^{b} e^{-\lambda(\xi-x)} f(\xi) d \xi\right]=\frac{1}{\pi} \int_{-\infty}^{\infty *} \frac{d \xi}{x-\xi} \int_{\xi}^{b} e^{-\lambda(\eta-\xi)}(D f)(\eta) d \eta \\
& =\frac{1}{\pi}\left[\int_{-\infty}^{-b_{*}}+\int_{-b}^{b_{*}}+\int_{b}^{\infty *}\right] \frac{d \xi}{x-\xi} \int_{\xi}^{b} e^{-\lambda(\eta-\xi)}(D f)(\eta) d \eta \equiv i_{1}^{\prime}+i_{2}^{\prime}+i_{3}^{\prime} .
\end{aligned}
$$

The integral $i_{3}^{\prime}=0$ since $(D f)(\eta)=0$ for $\eta>b$. Consider the second integral, then we have

$$
i_{2}^{\prime}=\frac{1}{\pi} \int_{-b}^{b_{*}} \frac{d \xi}{x-\xi} \int_{\xi}^{b} e^{\lambda(\eta-\xi)} f(\eta) d \eta=\left(\mathscr{L}_{b} \mathcal{L}^{*}(\lambda)\right)[f], \quad|x|<b,
$$

where $\mathcal{L}^{*}$ is given in (5.41). Finally, for $i_{1}^{\prime}$ we have when $|x|<b$ that

$$
i_{1}^{\prime}=\frac{1}{\pi} \int_{-\infty}^{-b_{*}} \frac{e^{\lambda \xi} d \xi}{x-\xi} \int_{\xi}^{b} e^{-\lambda \eta} f(\eta) d \eta=\frac{1}{\pi} \int_{-\infty}^{-b} \frac{e^{\lambda \xi} d \xi}{x-\xi} \int_{-b}^{b} e^{-\lambda \eta} f(\eta) d \eta=\frac{1}{\pi} \int_{-\infty}^{-b} \frac{e^{\lambda \xi} d \xi}{x-\xi} L(-\lambda, f) .
$$

In (5.45), we have taken into account that $|x|<b$ and definition (5.28). Finally, we consider an integral term from (5.45) and transform it as

$$
\frac{1}{\pi} \int_{-\infty}^{-b} \frac{e^{\lambda \xi}}{x-\xi} d \xi=\frac{1}{\pi} \int_{-b}^{\infty} \frac{e^{-\lambda \eta}}{x+\xi} d \xi=e^{\lambda x} \frac{1}{\pi} \int_{x+b}^{\infty} \frac{e^{-\lambda(\tau)}}{\tau} d \tau=e^{\lambda x} \varepsilon_{1}(\lambda(x+b))=g_{+}(\lambda, x),
$$

with $\varepsilon_{1}$ and $g_{+}$being defined in (5.16) and (5.42), respectively. Collecting together (5.43) and (5.45), we get (5.39).

The lemma is shown. 
Corollary 5.9. The operator corresponding to the multiplier $\varkappa_{2}$ from (5.35) can be given in the following form:

$$
\int_{0}^{\alpha_{2}} a(-s)\left[\left(\mathscr{L}_{b} \mathcal{L}^{*}(\lambda s)\right)[f]+g_{+}(\lambda s, x) L(-\lambda s, f)\right] d s, \quad|x|<b .
$$

Collecting together the results of Lemmas 5.4-5.8 and Corollaries 5.7 and 5.9, we arrive at an important result that we formulate as a theorem below.

Theorem 5.10. The Possio equation for $0<M<1$ can be expressed in the form

$$
\begin{aligned}
& W_{a}(x, \tilde{\lambda})=\frac{\sqrt{1-M^{2}}}{2} \\
& \times\left\{\mathscr{\ell}_{b}[F(\cdot, \tilde{\lambda})]-\frac{1}{\sqrt{1-M^{2}}}\left[\left(\lambda \mathscr{\ell}_{b} \mathcal{L}(\lambda)\right)[F(\cdot, \tilde{\lambda})]-\lambda g_{-}(x, \lambda) L(\lambda, F(\cdot, \tilde{\lambda}))\right]\right. \\
& -\int_{0}^{\alpha_{1}} a(s)\left[\left(\lambda \mathscr{\ell}_{b} \mathcal{L}(\lambda s)\right)[F(\cdot, \tilde{\lambda})]-\lambda g_{-}(x, \lambda s) L(\lambda s, F(\cdot, \tilde{\lambda}))\right] d s \\
& \left.+\int_{0}^{\alpha_{2}} a(-s)\left[\left(\lambda \mathscr{\ell}_{b} \mathcal{L}^{*}(\lambda s)\right)[F(\cdot, \tilde{\lambda})]-\lambda g_{+}(x, \lambda s) L(-\lambda s, F(\cdot, \tilde{\lambda}))\right] d s\right\}, \quad|x|<b .
\end{aligned}
$$

We will modify (5.48) to a more conventional form using Tricomi-Söhngen result [26, 27].

Proposition 5.11. Let $\rho(x)=\sqrt{(b-x) /(b+x)},|x|<b$, and let $L_{2, \rho}(-b, b)$ and $L_{2, \rho^{-1}}(-b, b)$ be the two weighted Hilbert spaces with the weights $\rho$ and $\rho^{-1}$, respectively. Then, the "airfoil equation" can be given in the form

$$
f(x)=\frac{1}{\pi} \int_{-b}^{b_{*}} \frac{w(\xi)}{x-\xi} d \xi=\frac{1}{\pi} \int_{-b}^{b_{*}} \frac{v(\xi)}{x-\xi} \rho(\xi) d \xi \equiv \mathbb{H}[v]
$$

The operator $\mathbb{H}$ defined in (5.49) is a Hilbert space isomorphism of $L_{2, \rho}(-b, b)$ onto $L_{2, \rho^{-1}}(-b, b)$. The inverse operator $\mathbb{H}^{-1}$ is given by

$$
v(x)=\mathbb{H}^{-1}[f](x)=\frac{1}{\pi} \int_{-b}^{b_{*}} \frac{f(\xi)}{x-\xi} \rho^{-1}(\xi) d \xi, \quad f \in L_{2, \rho^{-1}}(-b, b) .
$$

Equation (5.50) represents the classical Söhngen inversion formula for the airfoil equation in the presence of the Kutta condition. Multiplying both sides of (5.50) by $\rho$, one obtains

$$
w(x)=v(x) \rho(x)=\frac{1}{\pi} \sqrt{\frac{b-x}{b+x}} \int_{-b}^{b_{*}} \sqrt{\frac{b+\xi}{b-\xi}} \frac{f(\xi)}{x-\xi} d \xi \equiv \tau[f], \quad f \in L_{2, \rho^{-1}}(-b, b) .
$$

Our goal is to apply the operator $\tau$ to both sides of (5.48) in order to reduce it to a form convenient to deal with.

Lemma 5.12. The operator $\tau$ can be applied to each term of the right-hand side of (5.48). 
Proof. To prove this lemma, it suffices to show that (5.48) can be represented in the form

$$
\frac{2}{\sqrt{1-M^{2}}} W_{a}(x, \lambda)=\mathscr{H}_{b}\left[G_{1}\right]+G(x, \lambda),
$$

with $G \in L_{p}(-b, b), p>4 / 3$, and $G_{1} \in L_{p}(-b, b), 1<p<2$. Let us consider the sum of the first, second, fourth, and sixth terms from the right-hand side of (5.48) and have

$$
F_{1}=\mathscr{L}_{b}[F]+\frac{1}{\sqrt{1-M^{2}}} \mathscr{H}_{b}[\lambda \mathcal{L}(\lambda) F]-\int_{0}^{\alpha_{1}} a(s) \mathscr{L}_{b}[\mathcal{L}(\lambda s) F] d s+\int_{0}^{\alpha_{2}} a(-s) \mathscr{L}_{b}\left[\lambda \mathcal{L}^{*}(\lambda s) F\right] d s .
$$

Let us show that we can change the order of integration and the Hilbert transformation in the last two terms of (5.53). We discuss only the first integral; the second can be treated in a similar way. In the expression $\int_{0}^{\alpha_{1}} a(s) \mathscr{d}_{b}[\mathcal{L}(\lambda s) F] d s$ there are two integrals with the inner integral being integral with "the star" and the outer integral being standard. Since $a$ is a continuous function and $F \in L_{p}(-b, b)$, that is, both integrals go over finite intervals, one can change the order of integration [27]. Thus,

$$
\int_{0}^{\alpha_{1}} a(s) \mathscr{L}_{b}[\mathcal{L}(\lambda s) F] d s=\mathscr{L}_{b}\left[\int_{0}^{\alpha_{1}} a(s) \mathcal{L}(\lambda s) F\right] d s .
$$

Therefore,

$$
F_{1}=\mathscr{L}_{b}\left[F-\frac{1}{\sqrt{1-M^{2}}} \lambda \mathcal{L}(\lambda) F-\int_{0}^{\alpha_{1}} a(s) \mathcal{L}(\lambda s) F d s+\int_{0}^{\alpha_{2}} a(-s) \mathcal{L}^{*}(\lambda s) F d s\right] .
$$

It can be easily shown that the Hilbert transform $\mathscr{H}_{b}$ in (5.55) is applied to a function from $L_{p}(-\infty, \infty)$ as long as $F \in L_{p}(-b, b)$.

Now we consider the third, fifth, and seventh terms in the right-hand side of (5.48). Since $L(\cdot, F)$ is a functional over $F$, to verify that the sum of those three terms belongs to $L_{p}(-b, b)$, it suffices to check that the two functions $g_{-}(\cdot, \lambda)$ and $g_{+}(\cdot, \lambda)$ belong to $L_{p}(-b, b)$. The latter fact follows immediately from the definitions (5.19) and (5.42), and from the properties of "exponential integral" $\varepsilon_{1}$ of (5.16).

The lemma is completely shown.

In what follows, we will apply the operator $\tau$ to both sides of (5.48). In particular, we will need the expressions and the properties for the functions $h_{-}$and $h_{+}$defined below as

$$
h_{-}(x, \lambda)=\tau\left[e^{b \lambda} g_{-}(\cdot, \lambda)\right], \quad h_{+}(x, \lambda)=\tau\left[e^{b \lambda} g_{+}(\cdot, \lambda)\right] .
$$

Evaluating $h_{-}$, we have

$$
\begin{aligned}
h_{-}(x, \lambda) & =\frac{1}{\pi} \sqrt{\frac{b-x}{b+x}} \int_{-b}^{b_{*}} \sqrt{\frac{b+\xi}{b-\xi}} \frac{g_{-}(\xi, \lambda) e^{b \lambda}}{\xi-x} d \xi \\
& =\frac{1}{\pi} \sqrt{\frac{b-x}{b+x}} \int_{-b}^{b_{*}} \sqrt{\frac{b+\xi}{b-\xi}} \frac{d \xi}{\xi-x} e^{(b-\xi) \lambda} \int_{1}^{\infty} \frac{e^{-(b-\xi) \lambda t}}{t} d t \\
& =\frac{1}{\pi} \sqrt{\frac{b-x}{b+x}} \int_{-b}^{b_{*}} \sqrt{\frac{b+\xi}{b-\xi}} \frac{d \xi}{\xi-x} \int_{1}^{\infty} \frac{e^{-(b-\xi) \lambda(t-1)}}{t} d t
\end{aligned}
$$


Change of the variable of integration in (5.57), $\sigma=(b-\xi)(t-1)$, yields

$$
\begin{aligned}
h_{-}(x, \lambda) & =\frac{1}{\pi} \sqrt{\frac{b-x}{b+x}} \int_{-b}^{b_{*}} \sqrt{\frac{b+\xi}{b-\xi}} \frac{d \xi}{\xi-x} \int_{0}^{\infty} \frac{e^{-\lambda \sigma}}{b-\xi+\sigma} d \sigma \\
& =\frac{1}{\pi} \sqrt{\frac{b-x}{b+x}} \int_{0}^{\infty} \frac{1}{b-x+\delta} \sqrt{\frac{2 b+\sigma}{\sigma}} e^{\lambda \sigma} d \sigma, \quad|x|<b .
\end{aligned}
$$

Similarly, it can be shown that

$$
h_{+}(x, \lambda)=\frac{1}{\pi} \sqrt{\frac{b-x}{b+x}} \int_{0}^{\infty} \frac{1}{b+x+\sigma} \sqrt{\frac{\sigma}{2 b+\sigma}} e^{\lambda \sigma} d \sigma .
$$

The following properties for $h_{-}$and $h_{+}$hold:

$$
\lim _{x \rightarrow b-0} h_{-}(x, \lambda)=1, \quad \lim _{x \rightarrow b-0} h_{+}(x, \lambda)=0 .
$$

Finally, we present the desired modification of the integral equation (5.48).

Theorem 5.13. The Possio integral equation can be written in the following form:

$$
\begin{aligned}
\frac{2}{\sqrt{1-M^{2}}} \tau\left[W_{a}\left(\cdot, \lambda^{\prime}\right)\right]= & {\left[1-\frac{1}{\sqrt{1-M^{2}}} \lambda \mathcal{L}(\lambda)\right] F\left(\cdot, \lambda^{\prime}\right)+\frac{\lambda}{\sqrt{1-M^{2}}} h_{-}(x, \lambda) e^{-b \lambda} L\left(\lambda, F\left(\cdot, \lambda^{\prime}\right)\right) } \\
& -\int_{0}^{\alpha_{1}} a(s)(\lambda \mathcal{L}(\lambda s))[F] d s+\int_{0}^{\alpha_{1}} a(s) \lambda h_{-}(\cdot, \lambda s) e^{-b \lambda s} L\left(\lambda s, F\left(\cdot, \lambda^{\prime}\right)\right) d s \\
& +\int_{0}^{\alpha_{2}} a(-s)\left(\lambda \mathcal{L}^{*}(\lambda s)\right)[F] d s+\int_{0}^{\alpha_{2}} a(-s) \lambda h_{+}(\cdot, \lambda s) e^{-b \lambda s} L\left(-\lambda s, F\left(\cdot, \lambda^{\prime}\right)\right) d s .
\end{aligned}
$$

\section{Concluding remarks}

We would like to emphasize that (5.61) is a complicated singular integral equation. In our forthcoming work, we will prove a unique solvability of (5.61) using a two-step procedure. In the first step, we prove that as $|\lambda| \rightarrow \infty$, the right-hand side of the integral operator of (5.61) splits into two distinct parts: one does not get smaller and another one asymptotically goes to zero in a Banach space equipped with a specific norm. Having such a behavior in mind, we consider the equation obtained from (5.61) if one keeps only the leading asymptotical parts of all terms as $|\lambda| \rightarrow \infty, \mathfrak{R} \lambda \geqslant \delta_{a} \gg 1$. It turns out that the new equation (that we will call the asymptotical version of the Possio equation) is a singular integral equation as well. This asymptotic version of the Possio equation is a singular equation in more than one sense. First, it is singular due to the fact that it contains a specific "inverse operator" to a finite Hilbert transformation $\mathscr{H}_{b}\left(\mathscr{\ell}_{b}\right.$ is a singular integral operator in $\left.L_{p}(-b, b), 1<p<2\right)$. Secondly, the aforementioned equation contains integral operators being different by their nature: Hilbert transformation, Volterra integral operator, and an integral operator with the degenerate kernel [28]. It is exactly the second reason that makes the problem difficult and nonstandard. 


\section{Acknowledgment}

The authors highly appreciate the partial support of the National Science Foundation (Grants nos. ESC-0400730 and DMS-0604842).

\section{References}

[1] C. Possio, "Aerodynamic action on oscillating profile in compressible fluid at subsonic velocities," L'Aerotechnica, vol. 18, no. 4, pp. 441-458, 1938.

[2] A. V. Balakrishnan and M. A. Shubov, "Asymptotic behaviour of the aeroelastic modes for an aircraft wing model in a subsonic air flow," Proceedings of The Royal Society of London Series A, vol. 460, no. 2044, pp. 1057-1091, 2004.

[3] M. A. Shubov, "Riesz basis property of mode shapes for aircraft wing model (subsonic case)," Proceedings of The Royal Society of London Series A, vol. 462, no. 2066, pp. 607-646, 2006.

[4] M. A. Shubov, "Flutter phenomenon in aeroelasticity and its mathematical analysis," Journal of Aerospace Engineering, vol. 19, no. 1, pp. 1-13, 2006.

[5] E. Reissner, "On the theory of oscillating airfoils of finite span in subsonic compressible flow," NACA Technical Note NACA-TN-1953, NACA-TR-1002, NASA Center, Cambridge, Mass, USA, 1953.

[6] C. E. Watkins, H. L. Runyan, and D. S. Woolston, "The kernel function of the integral equation relating the lift and downwash distributions of oscillating finite wings in subsonic flow," Tech. Rep. NACATN-3131, NASA Langley Research Center, Hampton, Va, USA, 1954.

[7] A. V. Balakrishnan, "The Possio integral equation of aeroelasticity theory," Journal of Aerospace Engineering, vol. 16, no. 4, pp. 139-154, 2003.

[8] A. V. Balakrishnan, "The Possio integral equation of aeroelasticity: a modern view," in System Modeling and Optimization, F. Ceragioli, A. Dontchev, H. Furuta, K. Marti, and L. Pandolfi, Eds., vol. 199 of IFIP International Federation for Information Processing, pp. 15-22, Springer, New York, NY, USA, 2006.

[9] R. L. Bisplinghoff, H. Ashley, and R. L. Halfman, Aeroelasticity, Dover, New York, NY, USA, 1996.

[10] M. Goland, "The flutter of a uniform cantilever wing," Journal of Applied Mechanics, vol. 12, no. 4, pp. 197-208, 1945.

[11] P. S. Beran, T. W. Strganac, K. Kim, and C. Nichkawde, "Studies of store-induced limit cycle oscillations using a model with full system nonlinearities," Nonlinear Dynamics, vol. 37, no. 4, pp. 323-339, 2004.

[12] D. Hodges and E. H. Dowell, "Nonlinear equations of motion for the elastic bending and torsion of twisted nonuniform rotor blades," Tech. Rep. NASA-TN-D-7818, NASA Ames Research Center, Moffett Field, Calif, USA, 1974.

[13] A. V. Balakrishnan, "Subsonic flutter suppression using self-straining actuators," Journal of the Franklin Institute, vol. 338, no. 2-3, pp. 149-170, 2001.

[14] M. A. Shubov and C. A. Peterson, "Asymptotic distribution of eigenfrequencies for a coupled EulerBernoulli and Timoshenko beam model," NASA Technical Publication NASA-CR-2003-212022, NASA Dryden Flight Research Center, Edwards, Calif, USA, 2003.

[15] I. E. Garrick, H. Ashley, D. B. Hanson, A. E. Perry, and R. H. Scanlan, "Critical essays," in A Modern View of Theodore Theodorsen, E. H. Dowell, Ed., AIAA, Washington, DC, USA, 1992.

[16] R. Clark, D. Cox, H. C. Curtiss Jr., et al., Eds., A Modern Course in Aeroelasticity, vol. 116 of Solid Mechanics and Its Applications, Kluwer Academic Publishers, Dordrecht, The Netherlands, 4th edition, 2004.

[17] M. A. Shubov, "Mathematical modeling and analysis of flutter in long span suspension bridges and in blood vessel walls," Journal of Aerospace Engineering, vol. 17, no. 2, pp. 70-82, 2004.

[18] M. A. Shubov, "Mathematical modeling and analysis of flutter in bending-torsion coupled beams, rotating blades, and hard disk drives," Journal of Aerospace Engineering, vol. 17, no. 2, pp. 56-69, 2004.

[19] M. J. Patil, D. H. Hodges, and C. E. S. Cesnik, "Nonlinear aeroelastic analysis of complete aircraft in subsonic flow," Journal of Aircraft, vol. 37, no. 5, pp. 753-760, 2000.

[20] M. J. Patil and D. H. Hodges, "On the importance of aerodynamic and structural geometrical nonlinearities in aeroelastic behavior of high-aspect-ratio wings," Journal of Fluids and Structures, vol. 19, no. 7, pp. 905-915, 2004.

[21] C. S. Ventres, "Shear flow aerodynamics: lifting surface theory," AIAA Journal, vol. 13, no. 9, pp. 1183$1189,1975$. 
[22] E. H. Dowell and M. R. Chi, "Variable thickness shear layer aerodynamics revisited," AIAA Journal, vol. 15, no. 5, pp. 745-747, 1977.

[23] M. H. Williams, M. R. Chi, C. S. Ventres, and E. H. Dowell, "Aerodynamic effects of inviscid parallel shear flows," AIAA Journal, vol. 15, no. 8, pp. 1159-1166, 1977.

[24] S. G. Mikhlin, Multidimensional Singular Integrals and Integral Equations, Pergamon Press, New York, NY, USA, 1965.

[25] N. N. Lebedev, Special Functions and Their Applications, Dover, New York, NY, USA, 1972.

[26] D. Porter and D. S. G. Stirling, Integral Equations: A Practical Treatment, from Spectral Theory to Application, Cambridge Texts in Applied Mathematics, Cambridge University Press, Cambridge, UK, 1990.

[27] F. G. Tricomi, Integral Equations, Dover, New York, NY, USA, 1985.

[28] R. Estrada and R. P. Kanwal, Singular Integral Equations, Birkhäuser, Boston, Mass, USA, 2000. 


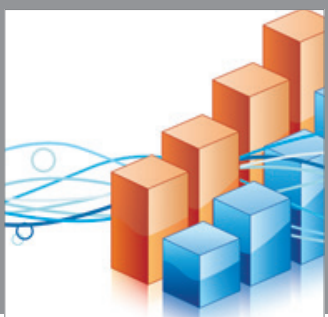

Advances in

Operations Research

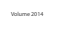

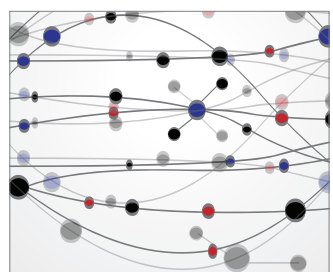

\section{The Scientific} World Journal
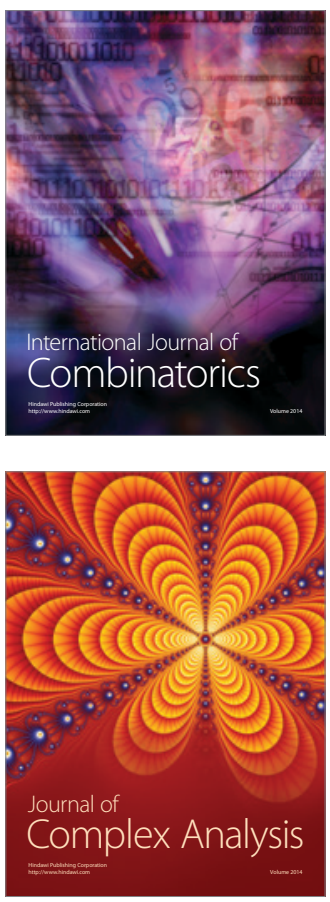

International Journal of

Mathematics and

Mathematical

Sciences
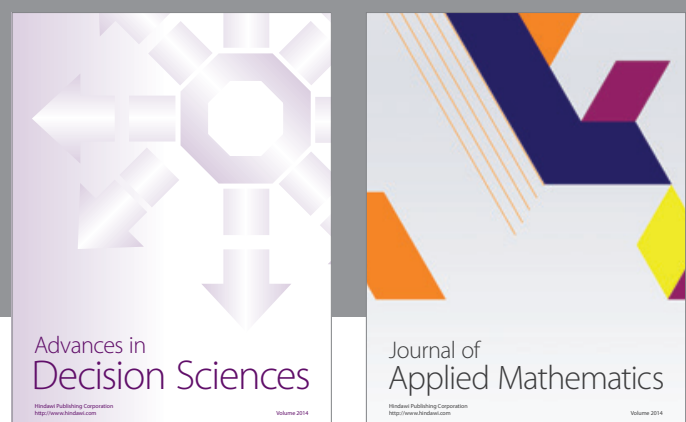

Journal of

Applied Mathematics
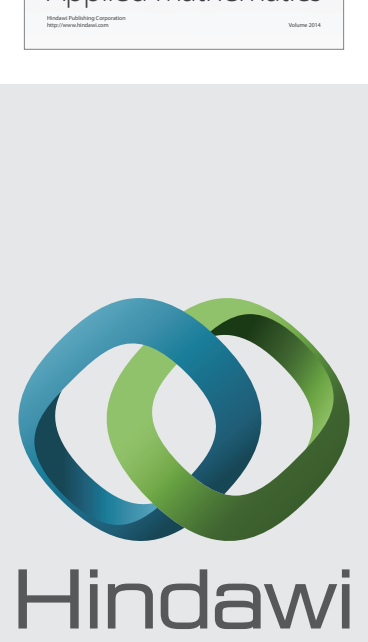

Submit your manuscripts at http://www.hindawi.com
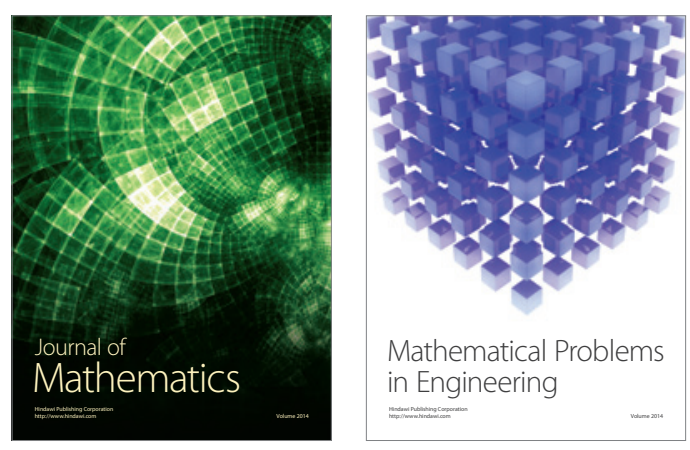

Mathematical Problems in Engineering
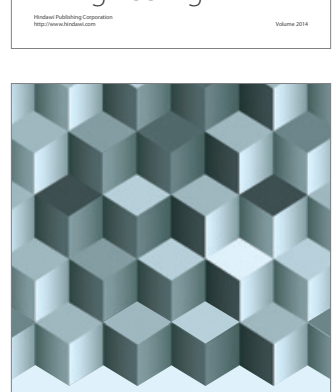

Journal of

Function Spaces
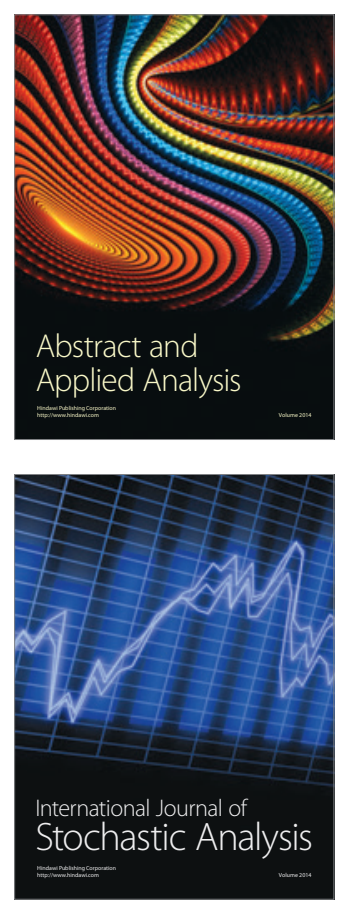

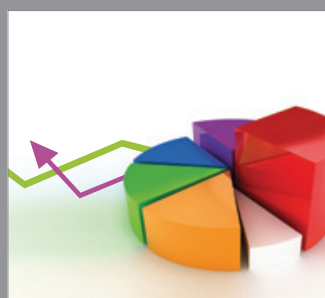

ournal of

Probability and Statistics

Promensencen
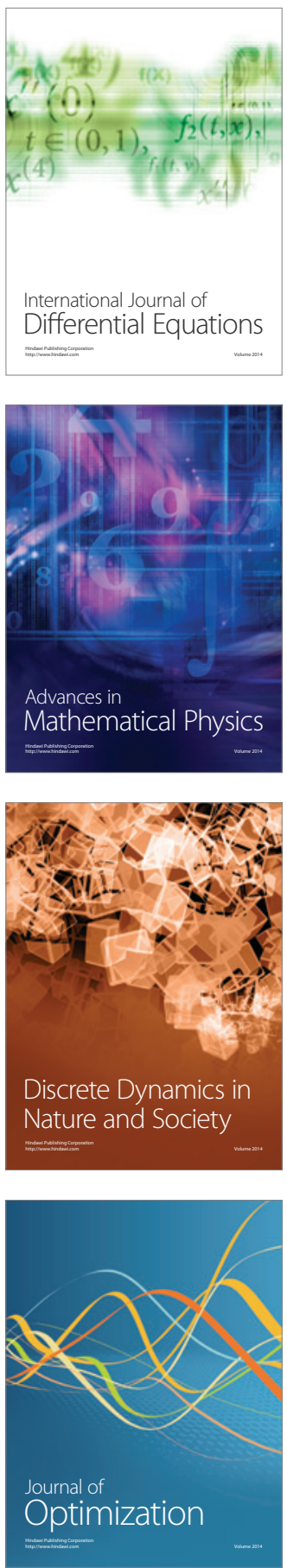